\begin{tabular}{|c|c|c|c|c|c|c|}
\hline \multirow{4}{*}{ Impact Factor: } & ISRA (India) & $=3.117$ & SIS (USA) & $=0.912$ & ICV (Poland) & $=6.630$ \\
\hline & ISI (Dubai, UAE & $=0.829$ & РИНЦ (Russia) & $=0.156$ & PIF (India) & $=1.940$ \\
\hline & GIF (Australia) & $=0.564$ & ESJI (KZ) & $=8.716$ & IBI (India) & $=4.260$ \\
\hline & JIF & $=1.500$ & SJIF (Morocco) & $=5.667$ & OAJI (USA) & $=0.350$ \\
\hline
\end{tabular}

\section{SOI: $1.1 /$ TAS $\quad$ DOI: $10.15863 /$ TAS \\ International Scientific Journal Theoretical \& Applied Science}

p-ISSN: 2308-4944 (print) e-ISSN: 2409-0085 (online)

Year: 2019 Issue: $07 \quad$ Volume: 75

Published: $30.07 .2019 \quad$ http://T-Science.org
QR - Issue

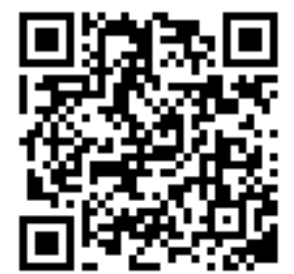

QR - Article

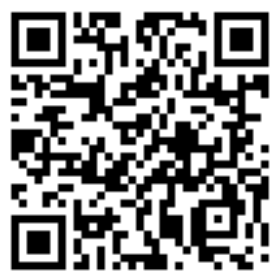

S. U. Zhanatauov

Noncommercial joint-stock company "Kazakh national agrarian university" Corresponding Member of International Academy of Theoretical and Applied Sciences (USA), Professor, Candidate of physics and mathematical sciences, Department «Information technologies and automatization», Kazakhstan sapagtu@mail.ru

\title{
COGNITIVE MODEL OF THE STRUCTURE OF THE MUNICIPAL BODY ON MONITORING THE MORAL ENVIRONMENT FOR SUBSIDES OF HUMAN RESOURCES
}

\footnotetext{
Abstract: The article presents mathematical and cognitive rationales for the establishment of 4 new Departments for monitoring the moral environment of human resources, in which it is advisable to include 12 specialists grouped by profiles from 2, 5, 1, 4 titles. The weights of the Departments are ordered in order of their priority: No. 1 (2 different profiles, 37.96\%), No. 2 (5 different profiles, 34.59\%), No. 3 (one profile, 34.157\%), No. 4 (4 different profiles, $25 \%$,). Specialists of different profiles should be combined into 5 subdivision, according to the number of expert values of the regression coefficients. Confirmatory calculations were carried out using simulation algorithms for the multidimensional $\left(\beta, C{ }^{+}{ }_{11}\right)$ sample of $z$-variables (with $\left.n=6\right)$. Descriptions of the coincidence of matrix equalities are given for the direct [1] process of mathematical introduction and for the reverse process of cognitive knowledge extraction.

Key words: new moral characteristics of the individual, mathematically introduced and cognitively extracted knowledge.

Language: Russian

Citation: Zhanatauov, S. U. (2019). Cognitive model of the structure of the municipal body on monitoring the moral environment for subsides of human resources. ISJ Theoretical \& Applied Science, 07 (75), 401-418.

Soi: http://s-o-i.org/1.1/TAS-07-75-66 Doi: crossef https://dx.doi.org/10.15863/TAS.2019.07.75.66

Classifiers: Applied mathematics. Mathematical modeling.

\section{КОГНИТИВНАЯ МОДЕЛЬ СТРУКТУРЫ МУНИЦИПАЛЬНОГО ОРГАНА ПО МОНИТОРИНГУ МОРАЛЬНОЙ СРЕДЫ ДЛЯ ПОДВИДОВ ЧЕЛОВЕЧЕСКИХ РЕСУРСОВ}

Аннотация: В статье приведены математические и когнитивные обоснования для учреждения 4-х новых Департаментов по мониторингу моральной среды человеческих ресурсов, в состав которых ичелесообразно включить 12 «узких» специиалистов, сгруппированных по профилям из 2-х,5-ти,1-го,4-х наименований. Веса Департаментов упорядочень в порядке их приоритетности: №1 (2 разных профиля, 37,96\%), №2 (5 разных профилей, 34.59\%), №3(один профиль, 34.157\%), №4 (4 разных профилей, 25\%,). Специалисты разных профилей должны быть объединены в 5 отделов-по числу экспертных значений коэффициентов регрессии. Подтверждаюшие расчеты проведены $с$ применением алгоритмов моделирования многомерной $\left(\boldsymbol{\beta}, C^{+}{ }_{11}\right)$-выборки z-переменных (при $\left.n=6\right)$. Использован назначенный экспертом вектор $\boldsymbol{\beta}=\left(\beta_{1} \ldots, \beta_{6}\right)^{T}$ регрессионных коэффициентов. Даны описания совпадения матричных равенств при прямом [1] прочессе математического введения и при обратном процессе когнитивного извлечения знаний.

Ключевые слова: новые моральные характеристики индивида, математически введенные и когнитивно извлекаемые знания.
} 


\begin{tabular}{|c|c|c|c|c|c|c|}
\hline \multirow{4}{*}{ Impact Factor: } & ISRA (India) & $=3.117$ & SIS (USA) & $=0.912$ & ICV (Poland) & $=6.630$ \\
\hline & ISI (Dubai, UAI & $=0.829$ & РИНЦ (Russia & $=0.156$ & PIF (India) & $=1.940$ \\
\hline & GIF (Australia) & $=0.564$ & ESJI (KZ) & $=8.716$ & IBI (India) & $=4.260$ \\
\hline & JIF & $=1.500$ & SJIF (Morocce & $=5.667$ & OAJI (USA) & $=0.350$ \\
\hline
\end{tabular}

\section{Введение}

Необходимо

существующих

благополучия», интегрированы в «международные витрины ресурсов и к таким подвидам, пользующиеся такими «средствами» как: связи, знакомства, рекомендации, знания, умения, навыки, репутация, присущи измеряемые моральные качества (показатели). Известно, что вышеназванные подвиды конвертируются друг в друга. «Например, мы с удовольствием инвестируем свое время для обретения новых связей и получения нового дохода. Когда мы нанимаем работников, мы покупаем за свои». Для достижения целей госуправления нужны ресурсы. Одними из их источников являются возможности граждан. Все ресурсы можно классифицировать по-разному. Мы рассматриваем некоторые из них, присущие трудовым ресурсам.

Индивидам, принадлежащим к группе человеческих ресурсов и к таким подвидам, пользующиеся такими «средствами» как: связи, знакомства, рекомендации, знания, умения, навыки, репутация, присущи измеряемые моральные качества (показатели). Известно, что вышеназванные подвиды конвертируются друг в друга. «Например, мы с удовольствием инвестируем свое время для обретения новых связей и получения нового дохода. Когда мы нанимаем работников, мы покупаем за свои деньги время других людей. Мы вкладываем деньги в обретение новых знаний, а знания конвертируем в навыки путем тренировок и применения на практике. А навыки, умело примененные приносят позитивную репутацию» ${ }^{1}$.

Приведенные ниже моральные показатели индивидов относятся к понятию "человеческие ресурсы", которое «более емкое, чем понятия "трудовые ресурсы" и "персонал", так как содержит в себе совокупность социокультурных характеристик и личностно-психологических свойств людей. «Специфика человеческих ресурсов в отличие от всех других видов ресурсов (материальных, финансовых, информационных и др.) состоит в следующем: а) люди наделены интеллектом, следовательно, их реакция на внешнее воздействие (управление) эмоциональноосмысленная, а не механическая; процесс взаимодействия между субъектом управления и людьми является двусторонним; б) вследствие обладания интеллектом люди способны к постоянному совершенствованию и развитию, что является наиболее важным и долговременным источником повышения эффективности любого общества или отдельной организации; в) люди выбирают определенный вид деятельности (производственный или непроизводственный, умственный или физический), осознанно ставя перед собой определенные цели. Поэтому субъект управления должен предоставлять все возможности для реализации этих целей, создавать условия для воплощения мотивационных установок к труду. Человеческие ресурсы являются конкурентным богатством любой организации. В 70-х гг. отделы кадров, отделы управления персоналом многих американских фирм и компаний переименованы в отделы. Человеческие ресурсы, где наряду с традиционными функциями (найм и отбор персонала, обучение, деловая оценка и др.) стали выполняться функции по стратегическому управлению человеческими ресурсами, формированию кадровой политики, разработке программ развития персонала, планированию потребности в человеческих ресурсах и т.п.» ${ }^{2}$

Премьер-министр Великобритании Тереза Мэй назначила министра по предотвращению само убийств. Она считает, что это должно поспособствовать уменьшению количества суицидов в стране. «10 октября в честь Всемирного дня психического здоровья в Лондоне прошел первый в мире саммит по вопросам психического здоровья, на котором были представители 50-ти стран. Именно на саммите Тереза Мэй назначила бывшего министр здраво охранения Джеки Дойла-Прайса на новую должность -министра по предотвращению самоубийств» ${ }^{3}$.

Также «премьер-министр Тереза Мэй объявила о назначении министра по вопросам одиночества. Согласно статистике, 4,5 тысячи человек в Великобритании каждый год лишают себя жизни. Хотя все же уровень самоубийств с каждым годом снижается» ${ }^{4}$.

Актуально иметь органы по мониторингу морально-психологической среды населения. Ниже излагается один из подходов формирования структуры муниципального органа по мониторингу моральной среды. Когнитивная модель структуры муниципальных органов по мониторингу моральной среды для видов человеческих ресурсов состоит из 4-х валидных моральных показателей, из 12 показателей, соответствующих своему валидному (и моральному) показателю. Среди 12 показателей имеются такие, которые имеют смысл одной zпеременной, входящей в несколько валидных упеременных $\mathrm{y}_{1}, \mathrm{y}_{2}, \mathrm{y}_{3}, \mathrm{y}_{4}$. Они могут иметь другой когнитивный смысл, например, $\mathrm{z}^{+}$-переменная №1
$1 \frac{\text { www.b17.ru/blog/78191 }}{\text { https://hr-portal.ru/varticle/chelovecheskie-resursy }}$
${ }^{3}$ www.kp.ua

4 www.tass.ru/obschestvo/4881148 


\begin{tabular}{|c|c|c|c|c|c|c|}
\hline \multirow{4}{*}{ Impact Factor: } & ISRA (India) & $=3.117$ & SIS (USA) & $=0.912$ & ICV (Poland) & $=6.630$ \\
\hline & ISI (Dubai, UAI & $=0.829$ & РИНЦ (Russia & $=0.156$ & PIF (India) & $=1.940$ \\
\hline & GIF (Australia) & $=0.564$ & ESJI (KZ) & $=8.716$ & IBI (India) & $=4.260$ \\
\hline & JIF & $=1.500$ & SJIF (Morocce & $=5.667$ & OAJI (USA) & $=0.350$ \\
\hline
\end{tabular}

$\left(\mathrm{z}^{+}{ }_{1}\right)$ входит в 2 валидные переменные $\mathrm{y}^{+}{ }_{2}, \mathrm{y}^{+}$. Мы придали ей один смысл, подчиняясь математическому факту - $\mathrm{Z}^{+}$-переменная $\mathrm{Z}^{+}{ }_{1}$ входит как слагаемое один раз в формулах $\left.\mathrm{y}^{+}{ }_{\mathrm{ij}}=\mathrm{z}^{+}{ }_{\mathrm{i} 1} \mathrm{c}^{+}{ }_{1 \mathrm{j}}+\ldots+\mathrm{z}^{+}{ }_{\mathrm{i} 5} \mathrm{c}^{+}{ }_{5 \mathrm{j}}, \quad \mathrm{j}=1, \ldots, 5, \mathrm{i}=1, \ldots, 20\right)$. Но зависит от 2-х валидных переменных $\mathrm{y}^{+}, \mathrm{y}^{+}{ }_{3}$. Их

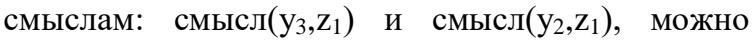
придать разные подсмыслы (Таблица, столбец 5, строки 2 и 3). С учетом смыслов таких $\mathrm{z}^{+}-$ переменных количество моральных показателей увеличится до 20. Для 12 или 20 показателей существует 6 показателей, обладающих суммарным смыслом той или иной части смысла вышеназванных 12 показателей. Они имеют 6 суммарных смыслов, каждый смысл порождает свое название отделу, с соответствующими функциональными обязанностями.

Ниже показаны обоснования для функционирования 4-х Департаментов мониторинга мораль ной среды у человеческих ресурсов, в состав которых целесообразно включить 12 узких специалистов, объединенных в 6 отделов. Число специалистов одного профиля (из 12) должно быть кратно фактической нагрузке по профилю. Вопросы нормирования труда каждого специалистов и вопросы оплаты их работы мы не рассматриваем.

Предлагаемая ниже переоценка показателей похожа на прогнозирование будущего (именно для этого чаще всего служит моделирование). Наше прогнозирование не есть восстановление прошлого, а является оцифровкой того, что есть в действительности, а не использование традиционных, не оправдывающих изменившиеся психологические, поведенческие доминанты.

Воспользуемся применениями валидных показателей, измеряющих то, что есть и трудно измеряемо, а не то, что удобно и легко измеряется.

Мы не рассматриваем такие моральные нормы, одобряемые и поощряемые обществом, даже находят своё отражение во многих законах различных государств. К типу таких моральных качеств относятся: совесть, достоинство, честь, справедливость. Мы рассматриваем другие моральные свойства: «по правде (правильно) поступать», «принудить к...», «обмануть, чтобы...», «страху нагнать». Они озвучиваются в обычной жизни простыми тружениками «на кухне» для сравнения индивидов по качествам. Они являются валидными показателями. Слово «валидные» имеет смысл «отражать действии тельность» (reflect, represent, validity, reality, permissibi lity, valid, effectivenes).

Рассматриваемые нами качества часто фигурируют у индивидов-экспертов в их умозаключениях. Мы называем их «моральные свойства», объявляем их валидными переменными, зависящими от других моделируемых z-переменных «» В первую очередь мы рассматривали не «моральные качества» (они измерялись в шкале отношений), а рассматривали измеряемые в шкале отношений «моральные свойства». Числовые значения некоторых из моральных показателей приведены в статье [1].

Цель исследования: выявление валидных и измеряемых показателей моральных качеств (из категории оппортунизма) через призму структуры муниципальных органов мониторинга человеческих ресурсов. Приоритетом при проектировании будущей структуры муниципальных органов мониторинга человеческих ресурсов по нашему мнению является моральная составляющая у их объектов мониторинга. Это придаст новый импульс работе социально ориентированных муниципальных органов, внедрит в жизнь достижения поведенческой экономики.

\section{Математические модели, когнитивные смыслы и смысловые задачи}

Мы используем модель цифровизации значений показателей индивидуального сознания, где решается Смысловая Обратная Задача (CO3) [2]. СО3 и задачи математического внесения индикаторов наличия знаний решались и их применения приведены в работах[3-6]. Смысловые обратные задачи [2] решаются при наличии математической модели и решаемых в ее рамках разных спектральных и оптимизационных задач [5-11]. Субъективно и когнитивно определяются когнитивные смыслы параметров и переменных математической модели, решаются смысловые прямые (СПЗ) и смысловые обратные задачи (СО3) [2,12-14]. Решениям Смысловых Задач предшествуют решения следующих математических задач из двух моделей. ОМ МЛРА[1,3-5] и ОМ ГК [12-14], Обратная Обобщенная Модель Множественной Линейной Регрессии (ООМ МЛРА) [1]. В ООМ МЛРА [1] решаются математические задачи ОС31 [11], ОС32 [9], Обратная Спектральная Задача для ООМ МЛРА[1,стр.616-620], Оптимизационная Задача [1,стр.616].

Суть нашего подхода может быть сведена не к анализу выборочных коэффициентов корреляции и коэффициентов регрессии. А может и должна содержать другие извлекаемые знания, кроме знаний, интерпретируемых из равенства $\mathrm{Z}_{2}=\mathrm{Z}_{1} \boldsymbol{\beta}$. Здесь [1] впервые рассматриваются элементы матрицы собственных векторов для моделирования влияющих на вектор $\boldsymbol{\beta}=\mathrm{R}^{-1}{ }_{11} \mathrm{R}_{12}$ подматриц. Решаются 3 OC3: ОС31 [11], ОС3 2 [9] ОС3 для ООМ МЛРА[1]. Эти 3 задачи решаются, если в матрицу $\mathrm{C}^{+} 11$ собственных векторов математически введены - через индикаторы наличия знаний, значения весомых «весов». 


\begin{tabular}{|c|c|c|c|c|c|c|}
\hline \multirow{4}{*}{ Impact Factor: } & ISRA (India) & $=3.117$ & SIS (USA) & $=0.912$ & ICV (Poland) & $=6.630$ \\
\hline & ISI (Dubai, UAI & $=0.829$ & РИНЦ (Russia & $=0.156$ & PIF (India) & $=1.940$ \\
\hline & GIF (Australia) & $=0.564$ & ESJI (KZ) & $=8.716$ & IBI (India) & $=4.260$ \\
\hline & JIF & $=1.500$ & SJIF (Morocce & $=5.667$ & OAJI (USA) & $=0.350$ \\
\hline
\end{tabular}

Полученные модельные матрицы данных $\mathrm{Z}_{\mathrm{mn}}=\left[\mathrm{Z}_{1} \mid \mathrm{Z}_{2}\right]$, будут иметь заданные целевые значения коэффициентов регрессии $\beta_{1}, \ldots, \beta_{\mathrm{n}-1}$, таких что $\mathrm{Z}_{2}=\mathrm{Z}_{1} \boldsymbol{\beta}$. Такое проектирование рядов векторов значений коэффициентов регрессии $\boldsymbol{\beta}=\left(\beta_{1}, \ldots, \beta_{\mathrm{n}-1}\right)^{\mathrm{T}}$ и соответствующих им рядов матриц $\quad\left\{\mathrm{Z}_{\mathrm{mn}}=\left[\mathrm{Z}_{1} \mid \mathrm{Z}_{2}\right]\right\} \quad$ с заданными алгебраическими и когнитивными свойствами позволяет через решение СПЗ и СО3, аналогичных приведенным в $[2,12-14]$ разработать Когнитивную Модель Структуры Муниципального Органа по Мониторингу Моральной Среды для подвидов Человеческих Ресурсов.

Извлечение введенных знаний (в ООМ МЛРА) проведем с применением соответствующей когнитивной модели, использующей математическую модель главных компонент [12-15]. Возможности соответствующих разным предметным областям когнитивных моделей показаны в публикациях [14-15].

\section{Программа -таблица моделирования матрицы $C^{(+)} 11$ и матрицы $\Lambda^{+}{ }_{11}$}

Кратко перечислим шаги вычислений нашей программы и приведем числовые значения элементов моделируемых и вычисляемых матриц (Таблица 1, Таблица 2, Таблица 3, Таблица 4, Таблица 5, Таблица 6). Они демонстрируют совпадение матриц, вычисленных в процессе моделирования, матрицам, вычисленным в процессе контроля правильности вычислений $\mathrm{Z}^{+}{ }_{20,6}=\left[\mathrm{Z}^{+}{ }_{1} \mid \mathrm{Z}_{2}\right]=>\left(\boldsymbol{\beta}, \Lambda^{+}{ }_{11}, \mathrm{C}^{+}{ }_{55}, \quad \beta^{+}{ }_{1}, \beta^{+}{ }_{2}, \beta^{+}{ }_{3}, \beta^{+}{ }_{4}\right.$, $\left.\beta^{+}{ }_{5}\right)$, где у модельной $\left(\boldsymbol{\beta}, \mathrm{C}^{+}{ }_{55}\right)$-выборки $\mathrm{Z}_{2}=\mathrm{Z}^{+}{ }_{1} \boldsymbol{\beta}$ или $\mathrm{Z}_{2}^{+}=\mathrm{Z}^{+}{ }_{1} \boldsymbol{\beta}^{+}$. Заданные значения $\beta_{1}=4, \beta_{2}=1$, $\beta_{3}=5, \beta_{4}=2, \beta_{5}=3$ в точности равны вычисленным (предсказанным по процедура Регрессия из надстройки Анализ данных ЭТ Excel) значениям (Таблица 3) нестандартизованной $z$-переменной $\mathrm{z}_{6}=\beta_{1} \mathrm{Z}^{+}{ }_{1}+\beta_{2} \mathrm{Z}^{+}{ }_{2}+\ldots+\beta_{5} \mathrm{Z}^{+}$, при вновь вычисленных значениях $\beta_{1}=4, \beta_{2}=1, \beta_{3}=5, \beta_{4}=2, \beta_{5}=3$ (Таблица 8).

При $\varphi=0.6$ мы смоделировали при решении Оптимизационной Задачи (Таблица 1, Рисунок 1 , Рисунок 2) один спектр из бесконечного множества спектров. Один из этих спектров: $\Lambda^{+}{ }_{11}=\operatorname{diag}(1,897809,1,127819,0,7923195,0,6451315$, 0,5369217) мы вычислили (Таблица 1), применяя процедуру Solver (Надстройка «Поиск решения» в ЭТ Excel). Работа с процедурой Solver состоит из следующих шагов [1].

Введем в ячейки ЭТ Excel известные начальные значения элементов матриц $\mathrm{C}_{11}$ и $\Lambda_{11}$ [1]. Проверяем в программе-таблице (Таблица 2) выполнение и точность равенств $\mathrm{C}^{\mathrm{T}}{ }_{11} \mathrm{C}_{11}=\mathrm{C}_{11} \mathrm{C}^{\mathrm{T}}{ }_{11}=\mathrm{I}_{(\mathrm{n}-1)(\mathrm{n}-1),} \mathrm{n}=6, \quad \mathrm{R}_{11} \mathrm{C}_{11}=\mathrm{C}_{11} \Lambda_{11}$.Эти значения являются начальными для реализации метода GRD2 в процедуре Solver. Далее реализуем ниже приведенные Шаги 1-7.

Шаг 1. Выделяем индексы $(\mathrm{k}, \mathrm{j})$ и значения элементов $\mathrm{c}^{+}{ }_{\mathrm{kj}}$ из матрицы $\mathrm{C}^{+}{ }_{11}$ c выделенными индексами Так как n-1=5, то введем пары ячеек ЭТ Excel назначенные значения элементов из каждого столбца матрицы $\mathrm{C}^{+}{ }_{55}$. Эти элементы не изменяют своих значений при нажатии кнопки «Выполнить» (Рисунок 1).

Шаг 2. Ввод в ячейки ЭТ Excel скалярных равенств, взятых из матричных равенств $\mathrm{C}^{+\mathrm{T}}{ }_{55} \mathrm{C}^{+}{ }_{55}=\mathrm{C}^{+}{ }_{55} \mathrm{C}^{+\mathrm{T}}{ }_{55}=\mathrm{I}_{55}, \quad \mathrm{R}^{+}{ }_{55}=\mathrm{C}^{+}{ }_{55} \Lambda^{+}{ }_{55} \mathrm{C}^{+\mathrm{T}}{ }_{55} . \quad$ Для элементов моделируемого нового спектра ${ }_{66} \Lambda^{+}{ }_{55}=\operatorname{diag}\left(\lambda^{+}{ }_{1}, \ldots, \lambda^{+}{ }_{5}\right) \quad$ введем ограничения $\lambda^{+}{ }_{1}+\ldots+\lambda^{+}{ }_{5}=5, \quad \lambda^{+}{ }_{1}>\ldots>\lambda^{+}{ }_{5}>0, \quad \lambda^{+}{ }_{1}=\lambda_{1}, \quad$ где $\lambda_{1-}$ известный всегда наибольший элемент известного спектра $\Lambda_{55}=\operatorname{diag}\left(\lambda_{1}, \ldots, \lambda_{5}\right)$.

Шаг 3. Назначить ячейку с формулой $\lambda^{+}{ }_{1}+\ldots+\lambda^{+}{ }_{5}$ целевой функцией процедуры Solver.

Шаг 4. Назначить в качестве изменяемых ячеек (неизвестных переменных задачи) ячейки матрицы $\mathrm{C}_{55}$ и ячейки элементов столбцов №1,...№5 을ицы $\mathrm{C}^{+} 55$.

Этим действием достигается неизменяемость компонентов собственных векторов №1, .,., №5.

Шаг 5. Назначить в качестве изменяемых еще 5 ячеек с значениями $\lambda^{+}{ }_{1} \ldots, \lambda^{+} 5$.

Шаг 6. Ввод в строке окна Solver ограничений для ячеек с их значениями вида $\lambda^{+}{ }_{1} \ldots>0, \ldots, \lambda_{5}{ }_{5}>0, \quad \lambda^{+}{ }_{1}=\lambda_{1}$. Ввод пороговых значений $1.05373033,0.08373033,0.70337303$, 0.001 для значений $\lambda^{+}{ }_{1} \ldots, \lambda^{+}{ }_{5}$, применявшихся для достижения строгих неравенств $\lambda^{+}{ }_{1}>\ldots>\lambda^{+}{ }_{5}>0.001$.

Шаг 7. Нажать кнопку «Выполнить».

Мы будем использовать решение ОС3 2, где найденная матрица не равна матрице $\mathrm{I}_{55}: \Lambda^{+}{ }_{55} \neq \mathrm{I}_{55}$ При этом выполняется соотношение $\Lambda^{+}{ }_{55}=\mathrm{C}^{+}$ ${ }_{55}^{\mathrm{T}} \mathrm{R}_{55}^{+} \mathrm{C}^{+}{ }_{55}, \mathrm{C}^{+}{ }_{55}, \quad$ при $\Lambda^{+}{ }_{55} \neq \mathrm{I}_{55}, \quad \mathrm{R}^{+}{ }_{55} \neq \mathrm{I}_{55}$. Пара матриц $\left(\Lambda^{+}{ }_{55}=\mathrm{C}^{+} \quad{ }_{55}^{\mathrm{T}} \mathrm{R}_{55}^{+} \mathrm{C}^{+}{ }_{55}, \mathrm{C}^{+}{ }_{55}\right)$ важна при когнитивном извлечении знаний и заменяет собой одну матрицу $\mathrm{R}^{+}{ }_{55}$, при ненулевых значениях ее коэффициентов корреляций ${ }^{+}{ }_{\mathrm{ji}}=\mathrm{r}^{+}{ }_{\mathrm{ij}} \neq 0, \mathrm{i} \neq \mathrm{j}=1, \ldots, \mathrm{n}-1$, $\mathrm{i}=1, \ldots, \mathrm{n}-1 . \mathbf{c}_{\mathrm{i}}^{+} \Lambda^{+}{ }_{11} \mathbf{c}_{\mathrm{j}}^{+\mathrm{T}}=\mathrm{r}_{\mathrm{ij}}^{+}, \mathrm{r}^{+}{ }_{\mathrm{ji}}=\mathrm{r}^{+}{ }_{\mathrm{ij}}, \mathrm{j}=1, \ldots, \mathrm{n}-1$.

Здесь при переходе от матрицы $\mathrm{R}^{+}{ }_{55}$ к паре матриц $\left(\Lambda^{+}{ }_{55}, \mathrm{C}^{+}{ }_{55},\right)$ можно применить термин «сверление» (dryll down). В информационных технологиях SAS Institute имеются системы с встроенными методами Drill down ("сверление"), "сенсоры" и "цветовая раскраска", помогающие пользователям ориентироваться в сложных данных, отслеживать тренды и зависимости и определять ключевые параметры.

Мы не используем свойство коэффициента $\mathrm{r}^{+}{ }_{\mathrm{ji}}$ как коэффициента линейной пропорциональности [16] между изменчивостью $\mathrm{z}_{\mathrm{ki}} \mathrm{i}-$ ой $\mathrm{z}$-переменной и изменчивостью $\mathrm{z}_{\mathrm{kj}} \mathrm{j}$-ой $\mathrm{z}-$ переменной: $\mathrm{z}_{\mathrm{ki}}=\mathrm{r}^{+}{ }_{\mathrm{ij}} \mathrm{z}_{\mathrm{kj}}, \mathrm{k}=1, \ldots, \mathrm{m}$. Мы используем другой коэффициент комбинационной связи [16] - 


\begin{tabular}{|c|c|c|c|c|c|c|}
\hline \multirow{4}{*}{ Impact Factor: } & ISRA (India) & $=3.117$ & SIS (USA) & $=0.912$ & ICV (Poland) & $=6.630$ \\
\hline & ISI (Dubai, UAI & $=0.829$ & РИНЦ (Russia & $=0.156$ & PIF (India) & $=1.940$ \\
\hline & GIF (Australia) & $=0.564$ & ESJI (KZ) & $=8.716$ & IBI (India) & $=4.260$ \\
\hline & JIF & $=1.500$ & SJIF (Morocce & $=5.667$ & OAJI (USA) & $=0.350$ \\
\hline
\end{tabular}

коэффициент $(\mathrm{y}, \mathrm{z})$-корреляций $\operatorname{corr}\left(\mathrm{y}_{\mathrm{i}}, \mathrm{z}_{\mathrm{j}}\right)=\mathrm{c}_{\mathrm{ij}}$. Эти равенства применимы для ненулевых значений $\mathrm{r}_{\mathrm{ji}}^{+} \neq 0$.

Моделируем нужную нам пару матриц $\left(\mathrm{C}^{+}{ }_{11}, \Lambda^{+}{ }_{11}\right)$, вместо схемы $\left(\mathrm{n}, \varphi_{11}\right)=>\left(\mathrm{R}_{11}\right)$ в ПМ МЛРА мы реализовали [19] схему $\left(\mathrm{n}, \varphi_{11}\right)=>\left(\mathrm{C}^{+}{ }_{11} \Lambda^{+}{ }_{11}\right)$. В матрицу $\mathrm{C}^{+}{ }_{11}$ мы вводим знания. Вложение знаний в матрицу $\mathrm{R}^{+}$ происходит через знания из известного вектора коэффициентов регрессии $\boldsymbol{\beta}$ и знания из модельной $\quad$ матрицы $\quad \mathrm{R}^{+}{ }_{11}=\mathrm{C}^{+}{ }_{11} \Lambda^{+}{ }_{11} \mathrm{C}^{+\mathrm{T}}{ }_{11}$. $\mathrm{R}^{(+)}{ }_{12}=\mathrm{R}^{(+)}{ }_{11} \beta$.

Этот новый вектор $\beta^{+}=\mathrm{R}^{+-1}{ }_{11} \mathrm{R}^{+}{ }_{12}=\mathrm{C}^{+}{ }_{11} \Lambda^{+[-}$ ${ }_{11}^{11} \mathrm{C}^{+} \mathrm{T}_{11} * \mathrm{R}^{+}{ }_{12}$ отличается от старого вектора $\beta=\mathrm{R}^{-}$ ${ }_{11}^{1} \mathrm{R}_{12}$, тем, что его компоненты зависят от матрицы $\mathrm{C}^{+}{ }_{11}$, содержащей нами введенные знания. Индикаторы присутствия знаний были заложены в нее. Зависящая от матрицы $\mathrm{C}^{+}{ }_{11}$ корреляционная матрица $\quad \mathrm{R}^{+}{ }_{11}=\mathrm{C}^{+}{ }_{11} \Lambda^{+}{ }_{11} \mathrm{C}^{+\mathrm{T}}{ }_{11}$ теперь также является $\mathrm{C}^{+}{ }_{11}$-источником знаний. Ограниченных знаний, как мы убедились на примере. Формула для подматрицы (вектора) $\mathrm{R}^{+}{ }_{12}$ в ООМ МЛРА принимает вид $\mathrm{R}^{+}{ }_{12}=\mathrm{R}^{+}{ }_{11} \boldsymbol{\beta}=\mathrm{C}^{+}{ }_{11} \Lambda^{+}{ }_{11} \mathrm{C}^{+\mathrm{T}}{ }_{11} \boldsymbol{\beta}$. И мы видим, что и под матрица $\mathrm{R}^{+}{ }_{11}$ и подматрица (вектор) $\mathrm{R}^{+}{ }_{12}$ зависят от $\mathrm{C}^{+}{ }_{11}$, содержащей математически введенные знания [1].

Теперь ОМ МЛРА изобразим по-другому, показывая матрицу $\mathrm{C}^{+}{ }_{11}: \quad\left(\mathrm{R}^{+}{ }_{11}, \mathrm{C}^{+}{ }_{11}, \Lambda^{+}{ }_{11}\right)=>$ $\left(\mathrm{Y}^{(\mathrm{t})}{ }_{\mathrm{m}(\mathrm{n}-1)}, \quad \mathrm{Z}^{(\mathrm{t})}{ }_{\mathrm{m}(\mathrm{n}-1)}\right), \mathrm{t}=1, \ldots, \mathrm{K}_{\mathrm{t}}<\infty, \quad$ а подзадачу 3 $\left(\mathrm{R}^{+}{ }_{11}, \beta\right)=>\mathrm{R}^{+}{ }_{12}$.

Результат решений трех подзадач обозначается в виде: $\left(\mathrm{m}, \mathrm{n}, \varphi_{11}, \boldsymbol{\beta}, \mathrm{C}^{+}{ }_{11}\right)=>\left(\mathrm{R}^{+}{ }_{11}, \mathrm{R}^{+}{ }_{12}, \mathrm{Z}^{+}{ }_{1}, \mathrm{Z}^{+}{ }\right)$.

Теоретические подробности и практические рекомендации по эксплуатации программытаблицы изложены в статье [1].

Необходимо во всех формулах проверять точность равенства левой части формулы ее правой части во всех матричных равенствах нашей модели. Например, по условию ОО3 МЛРА элементы из подматрицы $\mathrm{R}^{(\ell)}{ }_{12}=\mathrm{R}^{(\ell)}{ }_{11} \beta$ должны удовлетворять равенству $\mathrm{R}^{(\ell)}{ }_{12}=(1 / \mathrm{m}) \mathrm{Z}^{(\ell) \mathrm{T}}{ }_{1} \mathrm{Z}_{2}$. Это матричное уравнение должно точно выполняться численно. Таблицы числовых значений всех матриц из модели демонстрируют все матричные равенства. Эти же таблицы участвуют при обратном процессе извлечения введенных знаний с применением когнитивного моделирования.

\section{Когнитивные смыслы валидных и моделируемых показателей моральных качеств}

Смыслы моделируемых z-переменных, линейно входящих в валидные переменные $\mathrm{y}_{1}, \mathrm{y}_{2}$, $\mathrm{y}_{3}, \mathrm{y}_{4}$, не имеют смысловых противоречий. Только 2 z-переменные z,$z_{5}$ двояко (бинарно) именуются по смыслу. Речь идет об смыслах смысл $\left(\mathrm{y}_{1}, \mathrm{z}_{5}\right)=$ «честность» и смысл $\left(\mathrm{y}_{1}, \mathrm{z}_{3}\right)=$ порядочность. Назначение нами для пар смыслов («честность», «анти честность») пар $(\mathrm{y}, \mathrm{z})$-смыслов при $\mathrm{y}_{1} \neq \mathrm{y}_{4}$ : смысл $\left(\mathrm{y}_{1}, \mathrm{z}_{5}\right)=$ «честность» и смысл $\left(\mathrm{y}_{4}, \mathrm{z}_{5}\right)=$ анти честность, обосновано противоположностью знаков (+)-) при значениях коэффициентов $\mathrm{c}_{15}, \mathrm{c}_{45}$ : $\operatorname{corr}\left(\mathrm{y}_{1}, \mathrm{z}_{5}\right)=\mathrm{c}_{51}=0.5, \quad \operatorname{corr}\left(\mathrm{y}_{4}, \mathrm{z}_{5}\right)=\mathrm{c}_{54}=-0.5$. Аналогично обосновывается назначение смыслов в паре («порядочность», «антипорядочность»).

Наши 6 z-переменные $\mathrm{z}_{1}, \mathrm{z}_{2}, \mathrm{z}_{3}, \mathrm{z}_{4}, \mathrm{z}_{5}, \mathrm{z}_{6}$ заметно коррелированы попарно, а одна $z$-переменная $z_{6}$ линейно зависит от остальных $\mathrm{z}$-переменные $\mathrm{z}_{1}, \mathrm{z}_{2}$ $\mathrm{Z}_{3}, \mathrm{Z}_{4}, \mathrm{Z}_{5} \quad \mathrm{Z}_{6}=\beta_{1} \mathrm{Z}_{1}+\beta_{2} \mathrm{Z}_{2} \quad+\ldots+\beta_{5} \mathrm{Z}_{5}$.Матрицу $\mathrm{Z}^{+} \mathrm{z}_{\mathbf{1}}$ переменных $\mathbf{z}_{1}, \mathrm{z}_{2}, \mathrm{Z}_{3}, \mathrm{Z}_{4}, \mathrm{Z}_{5}$ мы смоделировали выше. Обсудим и экспертно назначим значения компонент для заданного нами вектора $\boldsymbol{\beta}$ и вычислим матрицу $\mathrm{Z}^{+}{ }_{2}=\mathrm{Z}^{+}{ }_{1} \boldsymbol{\beta}$.

\section{Назначенные и моделируемые значения регрессионных коэффициентов \\ Содержательный смысл $z$-переменных} $\mathrm{Z}_{1}, \mathrm{Z}_{2}, \mathrm{Z}_{3}, \mathrm{Z}_{4} \mathrm{Z}_{5}, \mathrm{Z}_{6}$ подсказывает нам: смысл $\left(\mathrm{y}_{3}, \mathrm{Z}_{6}\right)=$ «стремится обмануть, а не добросовестно сделать что-либо» образован из суммы смыслов 5 zпеременных $\mathrm{Z}_{1}, \mathrm{Z}_{2}, \mathrm{Z}_{3}, \mathrm{Z}_{4}, \mathrm{Z}_{5}$ и знаки при коэффициентах регрессии равны знаку «+». Вероятнее всего все знаки при коэффициенте $\boldsymbol{\beta}=\left(\beta_{1}, \ldots, \beta_{\mathrm{n}-1}\right)^{\mathrm{T}}$, имеют знак + . Каждое $\mathrm{j}$-ое значение $\left(\beta_{1}, \ldots, \beta_{\mathrm{n}-1}\right)^{\mathrm{T}}$ равно значению приращения к $\mathrm{F}\left(\mathrm{Z}_{6}+1\right)$ при увеличении на +1 значения j-ой $\mathrm{z}$-переменной $\mathrm{z}$ j: $\mathrm{F}\left(\mathrm{z}_{6}+1\right)=\mathrm{F}\left(\mathrm{z}_{6}\right)+\beta_{\mathrm{j}}$. Упорядочим по убыванию наши субъективные значения $\beta_{1}, \ldots, \beta_{5}$. Самым большим назначим значение $\beta_{3}$. Из всех смыслов мы считаем большим по весу «антипорядочность», а самым малым - значение $\beta_{2}$, ибо «низкая зарплата» при высокой безработице стало обычным явлением в Республике Казахстан. Остальные значения $\beta_{3}, \beta_{1}, \beta_{4}, \beta_{5}$, упорядочим аналогичным образом. Зафиксируем разницу между значениями наибольшего и наименьшего значений. После всех рассуждений и дискуссий имеем следующие значения $\beta_{1}, \ldots, \beta_{5}: \beta_{1}=4, \beta_{2}=1, \beta_{3}=5, \beta_{4}=2, \beta_{5}=3$. Это - назначенные значения. Далее вычисляем значения зависимой $\mathrm{z}-$-еременной $\mathrm{z}_{6}$ по формуле и нормируем значения $\mathrm{z}$-переменной $\mathrm{z}_{6}$ так, чтобы ее стандартное отклонение стало равным 1 . Условие равенства нулю ее средней арифметической выполняется автоматически. А ее стандартное отклонение не равно 1, ибо наши назначенные значения коэффициентам регрессии $\beta_{1}=4, \beta_{2}=1, \beta_{3}=5, \beta_{4}=2, \beta_{5}=3$ не соответствуют значениям стандартизованных $\mathrm{z}^{+}$-переменных. Они должны им соответствовать, но не соответствуют, так как ее значения назначены независимо от значений независимых $\mathrm{z}$ переменных. В ПЗ МЛРА значения коэффициентов регрессии зависят от значений $\mathrm{z}-$ 


\begin{tabular}{|c|c|c|c|c|c|c|}
\hline \multirow{4}{*}{ Impact Factor: } & ISRA (India) & $=3.117$ & SIS (USA) & $=0.912$ & ICV (Poland) & $=6.630$ \\
\hline & ISI (Dubai, UAI & $=0.829$ & РИНЦ (Russia & $=0.156$ & PIF (India) & $=1.940$ \\
\hline & GIF (Australia) & $=0.564$ & ESJI (KZ) & $=8.716$ & IBI (India) & $=4.260$ \\
\hline & JIF & $=1.500$ & SJIF (Morocce & $=5.667$ & OAJI (USA) & $=0.350$ \\
\hline
\end{tabular}

переменных, так как они вычисляются только если известна матрица значений всех 6 z-переменных. В нашей рассматриваемой О3 МЛРА матрица значений $\mathrm{Z}^{+}$-переменных неизвестна. Элементы матрицы значений $\mathrm{Z}_{20,6}^{+}=\left[\mathrm{Z}_{1}^{+} \mid \mathrm{Z}_{2}^{+}\right] \quad 6 \quad \mathrm{Z}^{+}-$ переменных мы моделируем по частям - сперва $\mathrm{Z}^{+}{ }_{1}$, потом - $\mathrm{Z}_{2}^{+}=\mathrm{Z}^{+} \boldsymbol{\beta}$. Так как при назначении значений коэффициентов регрессии $\beta_{1}, \ldots, \beta_{5}$ мы не учитывали их зависимость вида $\mathrm{Z}^{+}{ }_{2}=\mathrm{Z}^{+}{ }_{1} \boldsymbol{\beta}$ от значений $5 \mathrm{z}$-переменных $\mathrm{Z}_{1}, \ldots, \mathrm{z}_{5}$, то имеем другую зависимость вида $\mathrm{X}_{2}^{+}=\mathrm{Z}^{+}{ }_{1} \boldsymbol{\beta}$, где дисперсия $\mathrm{X}$-переменной не равна 1 : $\mathrm{s}_{\mathrm{x}}{ }^{2}=\left(\mathrm{x}^{2}{ }_{1 \mathrm{n}}+\mathrm{x}^{2}{ }_{2 \mathrm{n}}+\ldots+\mathrm{x}^{2}{ }_{\mathrm{mn}}\right) / \mathrm{m} \neq 1, \mathrm{n}=6, \mathrm{~m}=20$.

Стандартизацию значений х-переменной № 6 $(\mathrm{n}=6, \mathrm{~m}=20)$ проводим делением ее значений на число $\mathrm{s}_{\mathrm{x}}=0.09211$. Дисперсия новой $\mathrm{z}$-переменной теперь равна 1: $\mathrm{s}_{\mathrm{z}}{ }^{2}=\left(\mathrm{z}^{+2}{ }_{1 \mathrm{n}}+\mathrm{z}^{+2}{ }_{2 \mathrm{n}}+\ldots+\mathrm{z}^{+2}{ }_{\mathrm{mn}}\right) / \mathrm{m}=1$, $\mathrm{n}=6, \mathrm{~m}=20$. Эти стандартизованные значения $\mathrm{z}^{+}-$ переменной (расположены в столбце №6 матрицы $\mathrm{Z}^{+}{ }_{\mathrm{mn}}=\left[\mathrm{Z}^{+}{ }_{1} \int \mathrm{Z}^{+}{ }_{2}\right]$, Таблица 6 ,столбец №8) образуют подматрицу $\mathrm{Z}^{+}{ }_{2}=\left(\mathrm{Z}^{+}{ }_{1 \mathrm{n}}, \mathrm{Z}^{+}{ }_{2 \mathrm{n}}, \ldots, \mathrm{Z}^{+}{ }_{\mathrm{mn}}\right)^{\mathrm{T}}, \mathrm{n}=6$, удовлетворяющую соотношению $\mathrm{Z}^{+}{ }_{2}=\mathrm{Z}^{+}{ }_{1} \boldsymbol{\beta}$.

Наша программа-таблица обеспечивает совпадение значений элементов здесь смоделированных матриц с значениями элементов матриц, полученных при когнитивном моделировании извлечения введенных знаний, что будет видно, если вычислить по модельной матрице (Таблица 7). В Таблиц4 приведены вычисленные нестандартизованные значения Zпеременной $z_{6}$, вычисленные по формуле $\mathrm{z}_{6}=\beta_{1} \mathrm{Z}^{+}{ }_{1}+\beta_{2} \mathrm{z}^{+}{ }_{2}+\ldots+\beta_{5} \mathrm{Z}^{+}{ }_{5}$ из ОМ МЛРА (значения $\mathrm{Z}^{+}$- переменных $\mathrm{Z}^{+}{ }_{1}, \mathrm{Z}^{+}{ }_{2}, \ldots, \mathrm{z}^{+}{ }_{5}$ являются решениями ОСЗ для МЛРА). Нормированные значения $\mathrm{z}^{+}$переменной $\mathrm{z}_{6}^{+}$, представлены в 6-ом столбце матрицы (Таблица 6, столбец 8).

Таблица 1. Модельные значения элементов матрицы собственных векторов $\mathrm{C}^{+} 55$ и назначенные значения $\left|\mathbf{c}^{+}{ }_{\mathrm{kj}}\right|>\mathrm{c}_{\mathrm{j}}$ компонент собственных векторов

\begin{tabular}{|c|c|c|c|c|c|c|}
\hline & $\mathbf{c}_{1}$ & $\mathbf{c}_{2}$ & $\mathbf{c}_{3}$ & $\mathbf{c}_{4}$ & $\mathbf{c}_{5}$ & $c_{\text {i. }} c^{T_{i .}}=1$ \\
\hline & 1 & 2 & 3 & 4 & 5 & 6 \\
\hline ROW 1 & 0,3751292 & $-0,5000000$ & $-0,6500000$ & 0,0000000 & $-4,32 \mathrm{E}-01$ & 1,0000 \\
\hline ROW 2 & 0,5156684 & $-0,4000000$ & 0,2721876 & 0,5000000 & $5,00 \mathrm{E}-01$ & 1,0000 \\
\hline ROW 3 & $\mathbf{0 , 5 0 0 0 0 0 0}$ & $-0,5000000$ & 0,1205965 & $\mathbf{0 , 5 0 0 0 0 0 0}$ & $-4,85 \mathrm{E}-01$ & 1,0000 \\
\hline ROW 4 & 0,3055556 & $-0,5000000$ & 0,5606486 & $-0,5000000$ & $-3,04 \mathrm{E}-01$ & 1,0000 \\
\hline ROW 5 & 0,5000000 & 0,5000000 & $-0,1205965$ & $-0,5000000$ & $-4,85 \mathrm{E}-01$ & 1,0000 \\
\hline$c^{T_{j} c_{j}=1}$ & 1,0000 & 1,16000 & 0,8400 & 1,0000 & 1,0000 & \\
\hline$\Lambda_{11}^{+}$ & 1,8978 & 1,05373 & 1,05373 & 0,86461 & 0,13012 & 5 \\
\hline & 1 & 2 & 3 & 4 & 5 & \\
\hline$\Lambda_{11}$ & 1,8978 & 1,12782 & 0,79232 & 0,64513 & 0,53692 & 5,000001 \\
\hline \multicolumn{2}{|c|}{ Назначенные значения } & \multicolumn{5}{|c|}{$c^{+}{ }_{k j}>c_{j}, j=1,2,3,4,5$} \\
\hline$c_{51}$ & 0,5 & & & & & \\
\hline$c_{31}$ & 0,5 & & & & & \\
\hline$c_{12}$ & $-0,5000$ & & & & & \\
\hline $\mathbf{c}_{22}$ & $-0,4000$ & & & & & \\
\hline$c_{32}$ & $\mathbf{0 , 5 0 0 0}$ & & & & & \\
\hline$c_{42}$ & $-0,5000$ & & & & & \\
\hline$c_{52}$ & $\mathbf{0 , 5 0 0 0}$ & & & & & \\
\hline$c_{13}$ & $-0,6500$ & & & & & \\
\hline
\end{tabular}




\begin{tabular}{|c|c|c|c|c|c|c|}
\hline \multirow{4}{*}{ Impact Factor: } & ISRA (India) & $=3.117$ & SIS (USA) & $=0.912$ & ICV (Poland) & $=6.630$ \\
\hline & ISI (Dubai, UAI & $=0.829$ & РИНЦ (Russia & $=0.156$ & PIF (India) & $=1.940$ \\
\hline & GIF (Australia) & $=0.564$ & ESJI (KZ) & $=8.716$ & IBI (India) & $=4.260$ \\
\hline & JIF & $=1.500$ & SJIF (Morocce & $=5.667$ & OAJI (USA) & $=0.350$ \\
\hline
\end{tabular}

\begin{tabular}{||l|l||}
$\mathbf{c}_{24}$ & $\mathbf{0 , 5 0 0 0}$ \\
$\mathbf{c}_{34}$ & $\mathbf{0 , 5 0 0 0}$ \\
$\mathbf{c}_{44}$ & $\mathbf{- 0 , 5 0 0 0}$ \\
$\mathbf{c}_{54}$ & $\mathbf{- 0 , 5 0 0 0}$ \\
$\mathrm{C}_{25}$ & $\mathbf{0 , 5 0 0 0}$ \\
\hline
\end{tabular}

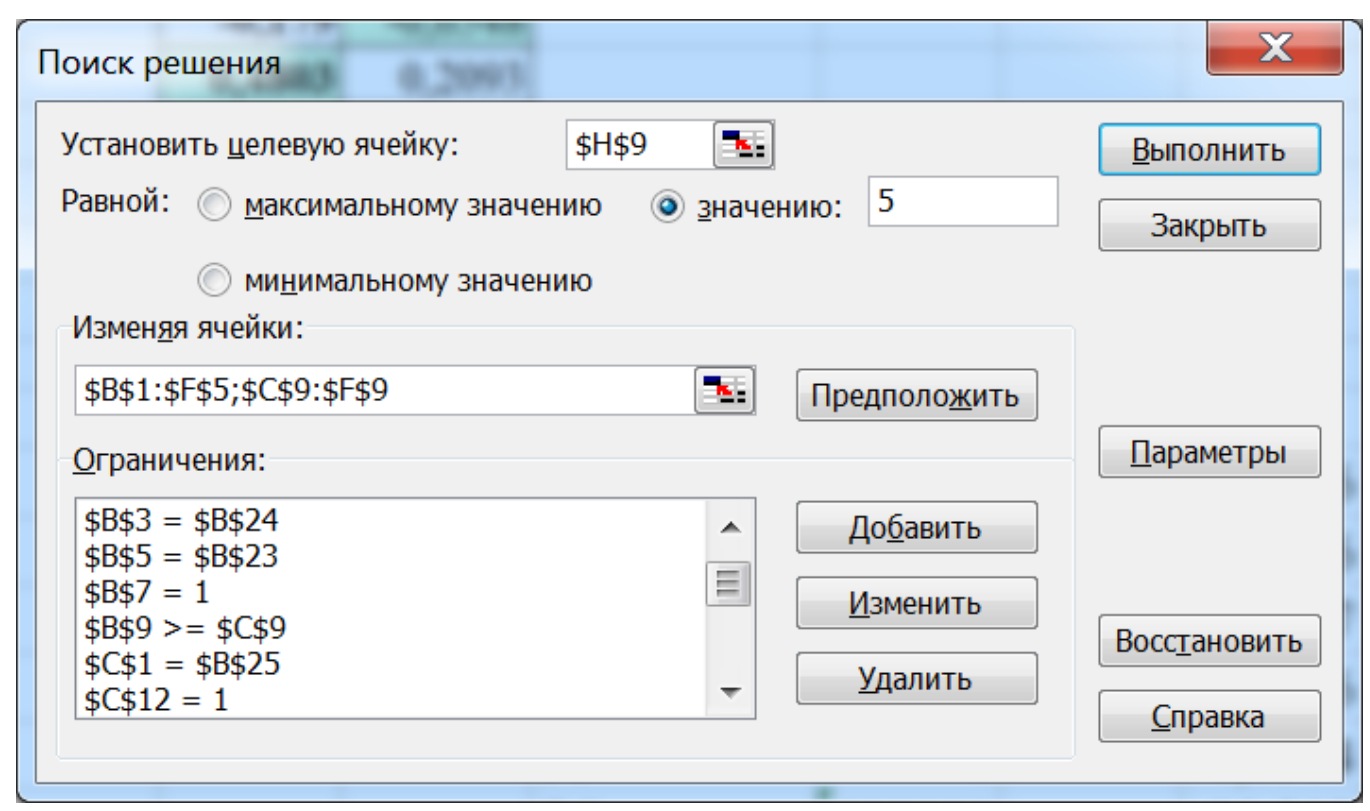

Рисунок 1. Окно процедуры «Поиск решения» в ЭТ Excel

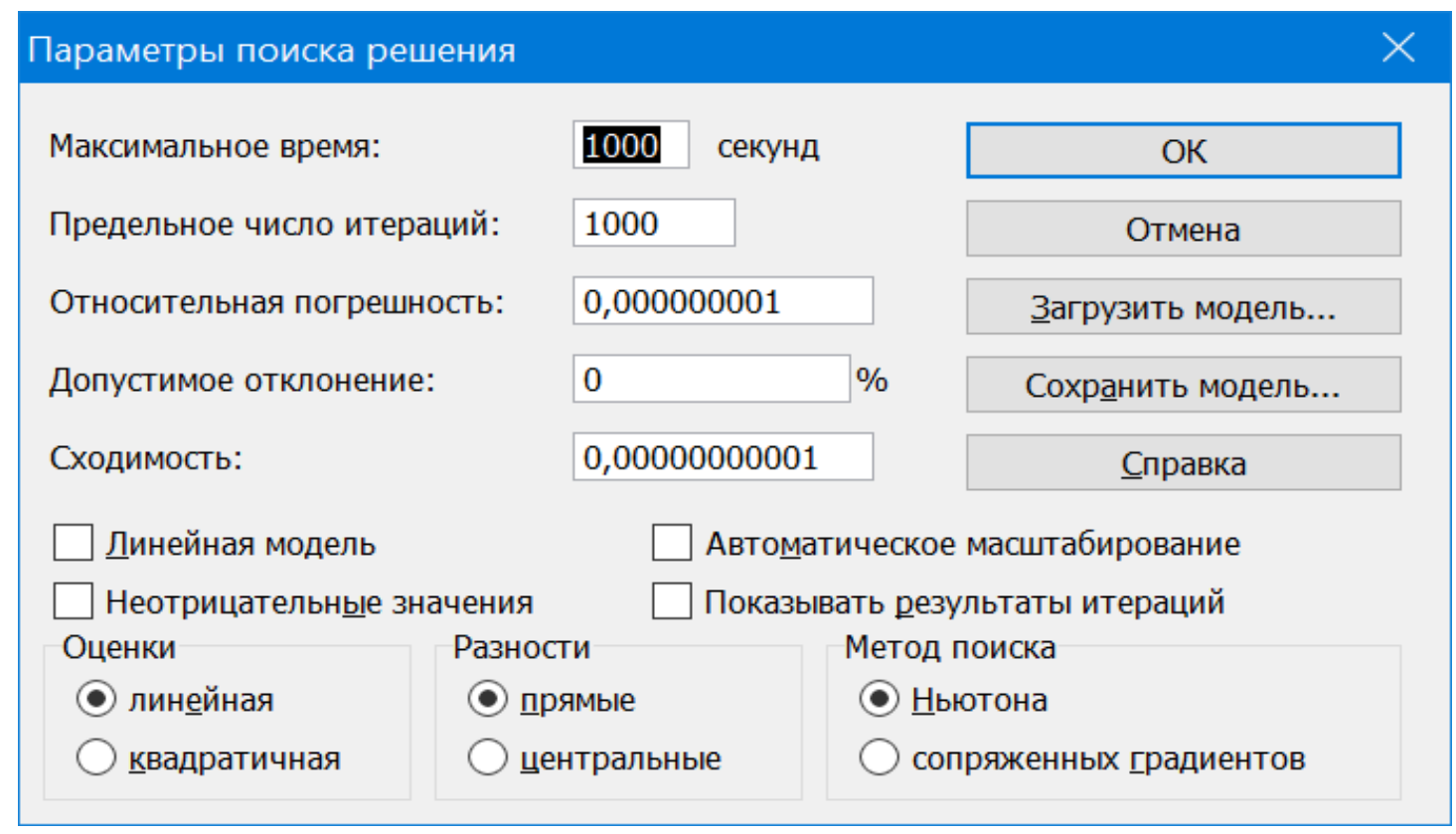

Рисунок 2. Значения параметров, применявшихся в процедуре «Поиск решения» в ЭТ ЕхсеІ 


\begin{tabular}{|c|c|c|c|c|c|c|}
\hline \multirow{4}{*}{ Impact Factor: } & ISRA (India) & $=3.117$ & SIS (USA) & $=0.912$ & ICV (Poland) & $=6.630$ \\
\hline & ISI (Dubai, UAI & $=0.829$ & РИНЦ (Russia & $=0.156$ & PIF (India) & $=1.940$ \\
\hline & GIF (Australia) & $=0.564$ & ESJI (KZ) & $=8.716$ & IBI (India) & $=4.260$ \\
\hline & JIF & $=1.500$ & SJIF (Morocce & $=5.667$ & OAJI (USA) & $=0.350$ \\
\hline
\end{tabular}

Таблица 2. Вычисленные значения коэффициентов регрессии $\beta=\left(\beta_{1}, \ldots, \beta_{5}\right)^{\mathrm{T}}$ для модельной $\left(\beta, C^{+}{ }_{55}\right)$ выборки $\left.Z^{+}{ }_{20,6}=\left[Z^{+}{ }_{1}\right] Z_{2}\right], Z_{2}=Z^{+} \beta$

ВЫВОД ИТОГОВ

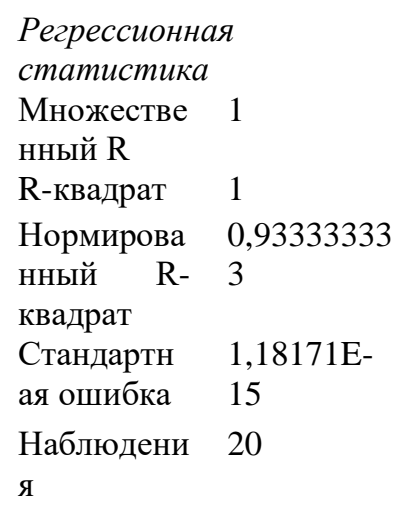

Дисперсионный анализ

\begin{tabular}{|c|c|c|c|c|c|c|c|c|}
\hline & $d f$ & $S S$ & $M S$ & $F$ & $\begin{array}{l}\text { Значимос } \\
\text { ть F }\end{array}$ & & & \\
\hline \multirow[t]{2}{*}{ Регрессия } & 5 & 2357,554 & 471,5108 & 3,37653 & $4,8013 \mathrm{E}-$ & & & \\
\hline & & 21 & 414 & $\mathrm{E}+32$ & 224 & & & \\
\hline Остаток & 15 & $\begin{array}{l}2,0947 \mathrm{E}- \\
29\end{array}$ & $\begin{array}{l}1,39644 \mathrm{E}- \\
30\end{array}$ & & & & & \\
\hline \multirow[t]{2}{*}{ Итого } & 20 & $\begin{array}{l}2357,554 \\
21\end{array}$ & & & & & & \\
\hline & $\begin{array}{l}\text { Коэффиии } \\
\text { ентьь }\end{array}$ & $\begin{array}{l}\text { Стандар } \\
\text { тная } \\
\text { Ошибка }\end{array}$ & $\begin{array}{l}t \text { - } \\
\text { статист } \\
\text { ика }\end{array}$ & $\begin{array}{l}P \text { - } \\
\text { Значени } \\
e\end{array}$ & $\begin{array}{l}\text { Нижние } \\
95 \%\end{array}$ & $\begin{array}{l}\text { Верхние } \\
95 \%\end{array}$ & $\begin{array}{l}\text { Нижние } \\
95,0 \%\end{array}$ & $\begin{array}{l}\text { Верx } \\
\text { ние } \\
95,0 \\
\%\end{array}$ \\
\hline
\end{tabular}

Y-

пересечени

e

Переменна 4

я z 1

Переменна 1

я z 2

Переменна 5

я z 3

Переменна 2

я z 4

Переменна 3

я z 5

$\begin{array}{llll}2,49728 \mathrm{E}- & 1,60174 \mathrm{E} & 1,1441 \mathrm{E}- & 4 \\ 15 & +15 & 220 & \\ 8,52559 \mathrm{E}- & 1,17294 \mathrm{E} & 1,2252 \mathrm{E}- & 1 \\ 15 & +14 & 203 & \\ 9,77468 \mathrm{E}- & 5,11525 \mathrm{E} & 3,1213 \mathrm{E}- & 5 \\ 15 & +14 & 213 & \\ 2,81212 \mathrm{E}- & 7,11207 \mathrm{E} & 2,2254 \mathrm{E}- & 2 \\ 15 & +14 & 215 \\ 2,29027 \mathrm{E}- & 1,30989 \mathrm{E} & 2,3379 \mathrm{E}- & 3 \\ 15 & +15 & 219 & \end{array}$

$\begin{array}{lll}4 & 4 & 4 \\ 1 & 1 & 1 \\ 5 & 5 & 5 \\ 2 & 2 & 2 \\ 3 & 3 & 3\end{array}$

Таблица 3. Вычисленные (предсказанные по ПМ МЛРА) значения нестандартизованной - z переменной $\mathrm{z}_{6}=\beta_{1} \mathrm{z}^{+}{ }_{1}+\beta_{2} \mathrm{z}^{+}{ }_{2}+\ldots+{ }_{5} \mathrm{Z}^{+}$, при $\beta_{1}=4, \beta_{2}=1, \beta_{3}=5, \beta_{4}=2, \beta_{5}=3$ (процедура Регрессия из надстройки Анализ данных ЭТ Excel)

\begin{tabular}{||l|l|l||}
\hline \hline \multicolumn{2}{|l||}{ ВЫВОД ОСТАТКА } \\
\hline 1 & 2 & 3 \\
\hline № наблюдения & Предсказанное Y & Остатки \\
\hline 1 & & \\
\hline 2 & $-7,4847500000$ & 0,0000000000 \\
\hline
\end{tabular}




\begin{tabular}{|c|c|c|c|c|c|c|}
\hline \multirow{4}{*}{ Impact Factor: } & ISRA (India) & $=3.117$ & SIS (USA) & $=0.912$ & ICV (Poland) & $=6.630$ \\
\hline & ISI (Dubai, UAE & $=0.829$ & РИНЦ (Russia) & $=0.156$ & PIF (India) & $=1.940$ \\
\hline & GIF (Australia) & $=0.564$ & ESJI (KZ) & $=8.716$ & IBI (India) & $=4.260$ \\
\hline & JIF & $=1.500$ & SJIF (Morocco) & $=5.667$ & OAJI (USA) & $=0.350$ \\
\hline
\end{tabular}

\begin{tabular}{||l|l|l||}
3 & $-4,6315300000$ & 0,0000000000 \\
\hline 4 & 8,7188615000 & 0,0000000000 \\
\hline 5 & 30,9865000000 & 0,0000000000 \\
\hline 6 & $-4,8289340000$ & 0,0000000000 \\
\hline 7 & $-0,3864900000$ & 0,0000000000 \\
\hline 8 & 2,4733100000 & 0,0000000000 \\
\hline 9 & 18,0439000000 & 0,0000000000 \\
\hline 10 & 3,1895400000 & 0,0000000000 \\
\hline 11 & 2,2764100000 & 0,0000000000 \\
\hline 12 & $-11,2819000000$ & 0,0000000000 \\
\hline 13 & $-14,2681100000$ & 0,0000000000 \\
\hline 14 & $-11,8772550000$ & 0,0000000000 \\
\hline 15 & $-7,7794300000$ & 0,0000000000 \\
\hline 16 & $-4,8824580000$ & 0,0000000000 \\
\hline 17 & $-7,7289500000$ & 0,0000000000 \\
\hline 18 & $-10,0166700000$ & 0,0000000000 \\
\hline 19 & 5,5096070000 & 0,0000000000 \\
\hline 20 & 10,7989400000 & 0,0000000000 \\
\hline \hline
\end{tabular}

Таблица 4. Вычисленные нестандартизованные значения z-переменной $\mathrm{z}_{6}$ (формула $\mathrm{z}_{6}=\beta_{1} \mathrm{z}^{+} 1+\beta_{2} \mathrm{z}^{+}{ }_{2}+\ldots+\beta_{5} \mathrm{z}^{+}$5из ОМ МЛРА, значения $\mathrm{z}^{+}$-переменных $\mathrm{z}^{+} 1, \mathrm{z}^{+} 2, \ldots, \mathrm{z}^{+}$я являются решениями ОСЗ для МЛРА)

\begin{tabular}{|c|c|c|c|c|c|c|c|c|c|c|}
\hline Наблюдение & 1 & 2 & 3 & 4 & 5 & 6 & 7 & 8 & 9 & 10 \\
\hline $\begin{array}{l}\text { модельноее } \\
\text { значение }\end{array}$ & $\begin{array}{l}- \\
7,484 \\
750\end{array}$ & $\begin{array}{l}3,1696 \\
35\end{array}$ & $\begin{array}{l}- \\
4,6315 \\
30\end{array}$ & $\begin{array}{l}8,7188 \\
62\end{array}$ & $\begin{array}{l}30,986 \\
500\end{array}$ & $\begin{array}{l}- \\
4,828 \\
934\end{array}$ & $\begin{array}{l}- \\
0,386 \\
490\end{array}$ & $\begin{array}{l}2,4733 \\
10\end{array}$ & $\begin{array}{l}18,043 \\
900\end{array}$ & $\begin{array}{l}3,1895 \\
40\end{array}$ \\
\hline Наблюдение & 11 & 12 & 13 & 14 & 15 & 16 & 17 & 18 & 19 & 20 \\
\hline $\begin{array}{l}\text { модельное } \\
\text { значение }\end{array}$ & $\begin{array}{l}2,276 \\
410\end{array}$ & $\begin{array}{l}- \\
11,281 \\
900\end{array}$ & $\begin{array}{l}- \\
14,268 \\
110\end{array}$ & $\begin{array}{l}- \\
11,877 \\
255\end{array}$ & $\begin{array}{l}- \\
7,7794 \\
30\end{array}$ & $\begin{array}{l}- \\
4,882 \\
458\end{array}$ & $\begin{array}{l}- \\
7,728 \\
950\end{array}$ & $\begin{array}{l}- \\
10,016 \\
670\end{array}$ & $\begin{array}{l}5,5096 \\
07\end{array}$ & $\begin{array}{l}10,798 \\
940\end{array}$ \\
\hline
\end{tabular}

Исходные заданные экспертом значения коэффициентов регрессии $\beta_{1}, \beta_{2}, \beta_{3}, \beta_{4}, \beta_{5}$ задают пропорциональные зависимости между их значениями. Это удобно эксперту, так как наличие равного 1 коэффициента, например, $\beta_{2}=1$, задает шаговое значение сравнения $\mathrm{c}$ другими значениями коэффициентов регрессии, больших 1. Другие значения коэффициентов регрессии дают назначенные экспертом аддитивные приращения, аддитивно отличающиеся от $\beta_{2}=1$, что соответствует содержательному смыслу значения коэффициента регрессии $\mathrm{F}\left(\mathrm{z}_{\mathrm{j}}+1\right)=\mathrm{F}\left(\mathrm{z}_{\mathrm{j}}\right)+\beta_{\mathrm{j}} . \quad$ Равенство $\quad \mathrm{F}\left(\mathrm{z}_{2}+1\right)=\mathrm{F}\left(\mathrm{z}_{2}\right)+\beta_{2}$ является «точкой отсчета», значения остальных коэффициентов регрессии пропорциональны значению $\beta_{2}=1$, они все больше 1. Ниже мы рассматриваем случай, когда $\beta_{2}=1$ является минимальным коэффициентом регрессии, дающим минимальное приращение значению зависимой z-переменной $\mathrm{z}_{6}: \mathrm{z}_{6}=\mathrm{F}\left(\mathrm{z}_{2}+1\right)=\mathrm{F}\left(\mathrm{z}_{2}\right)+\beta_{2}$.
Исходные значения $\beta_{1}=4, \beta_{2}=1, \beta_{3}=5, \beta_{4}=2$, $\beta_{5}=3$ удобны для конструировании фраз когнитивных смыслов, но они были преобразованы при нормировании z-переменной №6.

Нас интересует значение коэффициента преобразования одной совокупности коэффициентов регрессии в другую совокупность коэффициентов регрессии при стандартизованных значениях $\mathrm{Z}^{+}$-переменных $\mathrm{Z}^{+} 1, \mathrm{Z}^{+}, \ldots, \ldots, \mathrm{Z}^{+}$. Зная нашу модельную матрицу $\left[\mathrm{Z}^{+} 1 \mid \mathrm{Z}^{+}{ }_{2}\right], \mathrm{Z}^{+}{ }_{2}=\mathrm{Z}^{+}{ }_{1} \boldsymbol{\beta}$ мы вычислили коэффициенты регрессии $\beta^{+}{ }_{1}, \ldots, \beta^{+}$. Они отличаются от прежних коэффициентов регрессии. При этом использовали процедуру Regression в ЭТ Excel. Это является реализацией ПМ МЛРА, при этом, как ожидалось, получили те же матрицы $\mathrm{R}^{+}{ }_{11}$, $\mathrm{R}^{+}{ }_{12}, \mathrm{R}^{+}{ }_{22}=(1)$, определяемые по формулам $(1 \backslash 20) \mathrm{Z}_{1}{ }^{+\mathrm{T}} \mathrm{Z}^{+}{ }_{1}=\mathrm{R}^{+}{ }_{11},(1 \backslash 20) \mathrm{Z}_{1}{ }^{+\mathrm{T}} \mathrm{Z}^{+}{ }_{2}=\mathrm{R}_{12}$,

$(1120) \mathrm{Z}_{2}{ }^{+\mathrm{T}} \mathrm{Z}^{+}{ }_{2}=\mathrm{R}^{+}{ }_{22}=(1)$ и являются разбиениями 


\begin{tabular}{|c|c|c|c|c|c|c|}
\hline \multirow{4}{*}{ Impact Factor: } & ISRA (India) & $=3.117$ & SIS (USA) & $=0.912$ & ICV (Poland) & $=6.630$ \\
\hline & ISI (Dubai, UAI & $=0.829$ & РИНЦ (Russia & $=0.156$ & PIF (India) & $=1.940$ \\
\hline & GIF (Australia) & $=0.564$ & ESJI (KZ) & $=8.716$ & IBI (India) & $=4.260$ \\
\hline & JIF & $=1.500$ & SJIF (Morocce & $=5.667$ & OAJI (USA) & $=0.350$ \\
\hline
\end{tabular}

корреляционной матрицы $\mathrm{R}^{+}{ }_{66}=(1 \backslash 20) \mathrm{Z}^{+\mathrm{T}}{ }_{20,6 \mathrm{n}} \mathrm{Z}^{+}{ }_{20,6}$, соответствующих разбиению $\mathrm{Z}^{+}{ }_{\mathrm{mn}}=\left[\mathrm{Z}^{+} \mid \mathrm{Z}^{+}{ }_{2}\right]$.

Вычисленные значения регрессионных коэффициентов $\quad \beta^{+}{ }_{1}=0,368420804$, $\beta^{+}{ }_{2}=0,092105201, \quad \quad \beta^{+}{ }_{3}=0,460526005$, $\beta^{+}{ }_{4}=0,184210402, \quad \beta^{+}=0,276315603 \quad$ являются решением ПЗ МЛРА из ПМ МЛРА: $\mathrm{Z}^{+}{ }_{\mathrm{mn}}=\left[\mathrm{Z}^{+}{ }_{1} \mid \mathrm{Z}^{+}{ }_{2}\right]=>\left(\mathrm{R}^{+-1}{ }_{11}, \mathrm{R}^{+}{ }_{12}, \beta^{+}\right)$, где для уже известных модельных матриц реализована ПМ МЛРА: $\mathrm{Z}^{+}{ }_{\mathrm{mn}}=\left[\mathrm{Z}^{+}{ }_{1} \mid \mathrm{Z}^{+}{ }_{2}\right]=>\left(\mathrm{R}^{+-1}{ }_{11}, \mathrm{R}^{+}{ }_{12}, \beta^{+}\right), \quad$ Таким образом мы убедились в равенствах матриц $\mathrm{Z}^{+}{ }_{\mathrm{mn}}=\left[\mathrm{Z}^{+}{ }_{1}\right] \mathrm{Z}^{+}{ }_{2}, \mathrm{R}^{+-1}{ }_{11}, \mathrm{R}^{+}{ }_{12}, \beta^{+}$, численно вычисленных в ПМ МЛРА и в ОМ МЛРА.

Для нашего экспертного набора коэффициентов регрессии $\beta_{1}, \ldots, \beta_{5} \quad \beta_{1}=4, \beta_{2}=1$, $\beta_{3}=5, \beta_{4}=2, \beta_{5}=3$ и нового набора коэффициентов регрессии $\beta^{+}{ }_{1}, \ldots, \beta^{+}{ }_{5}$ мы нашли значение коэффициента преобразования 0.092105201 (Таблица 8)-оно является наименьшим коэффициентом регрессии $\beta^{+}{ }_{2}=\min \left\{\beta^{+}{ }_{1}, \ldots, \beta^{+}{ }_{5}\right\}$ $=0,092105201, \mathrm{j}=1, \ldots, 5$. Если нас устраивает набор коэффициентов регрессии $\beta_{1}, \ldots, \beta_{5}$ такой, что $\beta_{1}=4$, $\beta_{2}=1, \beta_{3}=5, \beta_{4}=2, \beta_{5}=3$, то в ОМ МЛРА им соответствует модельный набор коэффициентов регрессии $\beta^{+}{ }_{1}=\beta_{1} * \beta^{+}{ }_{2}=0.368420804 ; \beta^{+}{ }_{2}==\beta_{2} * \beta^{+}{ }_{2}$ $=0.092105201 ; \beta_{3}{ }_{3}=\beta_{3} * \beta^{+}{ }_{2}=0.460526005 ; \beta^{+}{ }_{4}=\beta_{4} * \beta^{+}{ }_{2}$ $=0.184210402 ; \beta^{+}{ }_{5}=\beta_{5} * \beta^{+}{ }_{2}=0.276315603$.

Мы облегчили для эксперта подбор значений коэффициентов регрессии. На примере показали, что для любого набора значений $\beta^{+}{ }_{1}, \ldots, \beta^{+}{ }_{5}$ (коэффициентов регрессии при стандартизованных $\mathrm{z}^{+}$-переменных $\mathrm{z}^{+}{ }_{1}, \mathrm{z}^{+}, \ldots, \mathrm{z}^{+}$) существует единственный набор «экспертных» коэффициентов регрессии $\beta_{1}, \ldots, \beta_{5}$ при смыслах независимых $\mathrm{z}^{+}$-переменных $\mathrm{z}^{+}, \mathrm{z}^{+}{ }_{2}, \ldots, \mathrm{z}^{+}{ }_{4}$, z5для смысла одной нестандартизованной зависимой $\mathrm{z}-$ переменной Z6.

Теперь после этапа математического введения знаний и этапа назначения желаемого набора коэффициентов регрессии $\beta^{+}{ }_{1}, \ldots, \beta^{+}{ }_{5}$ мы приступим к обратному процессу - к шагам этапа когнитивного извлечения введенных ранее знаний.

Таблица 5.

\begin{tabular}{|c|c|c|c|c|c|}
\hline & \multicolumn{5}{|c|}{ Значения коэффициентов регрессии $\beta_{1}, \beta_{2}, \beta_{3}, \beta_{4}, \beta_{5}$} \\
\hline 1 & 2 & 3 & 4 & 5 & 6 \\
\hline Заданные экспертом & $\beta_{1}=4$ & $\beta_{2}=1$ & $\beta_{3}=5$ & $\beta_{4}=2$ & $\beta 5=3$ \\
\hline 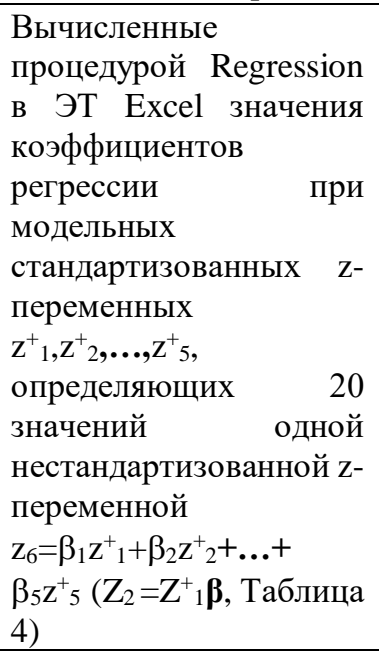 & $\beta_{1}=4$ & $\beta_{2}=1$ & $\beta_{3}=5$ & $\beta_{4}=2$ & $\beta=3$ \\
\hline 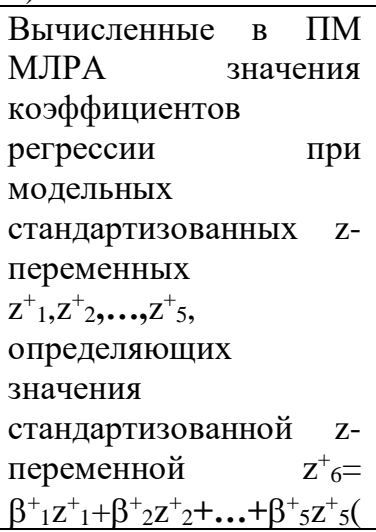 & $\begin{array}{l}\beta^{+}{ }_{1}=0,368420 \\
804\end{array}$ & $\begin{array}{l}\beta^{+}{ }_{2}=0,092105 \\
201\end{array}$ & $\begin{array}{l}\beta^{+}{ }_{3}=0,460526 \\
005\end{array}$ & $\begin{array}{l}\beta^{+}{ }_{4}=0,184210 \\
402\end{array}$ & $\begin{array}{l}\beta^{+}{ }_{5}=0,276315 \\
603\end{array}$ \\
\hline
\end{tabular}




\begin{tabular}{|c|c|c|c|c|c|c|}
\hline \multirow{4}{*}{ Impact Factor: } & ISRA (India) & $=3.117$ & SIS (USA) & $=0.912$ & ICV (Poland) & $=6.630$ \\
\hline & ISI (Dubai, UAE & $=0.829$ & РИНЦ (Russia) & $=0.156$ & PIF (India) & $=1.940$ \\
\hline & GIF (Australia) & $=0.564$ & ESJI (KZ) & $=8.716$ & IBI (India) & $=4.260$ \\
\hline & JIF & $=1.500$ & SJIF (Morocco) & $=5.667$ & OAJI (USA) & $=0.350$ \\
\hline
\end{tabular}

\begin{tabular}{|l|l|l|l|l|l|}
\hline $\begin{array}{l}\mathrm{Z}^{+}=\mathrm{Z}^{+}{ }_{1} \boldsymbol{\beta}^{+} \text {, Таблица 6, } \\
\text { столбец 8). }\end{array}$ & & & & \\
\hline $\begin{array}{l}\text { Формула связи } \beta^{+}{ }_{\mathrm{j}} \text { с } \beta_{\mathrm{j}} \\
\beta^{+}=\beta_{\mathrm{j}}{ }^{*} \min \left\{\beta^{+}{ }_{1}, \ldots, \beta^{+}{ }_{\mathrm{n}-}=\beta_{1} / \beta^{+}{ }_{2}=\right.\end{array}$ & 0,368420804 & $\beta^{+}{ }_{2}=\beta_{2} / \beta^{+}{ }_{2}=$ & $\beta^{+}{ }_{3}=\beta_{3} / \beta^{+}{ }_{2}=$ & $\beta^{+}{ }_{4}=\beta_{4} / \beta^{+}{ }_{2}=$ & $\beta^{+}{ }_{5}=\beta_{5} / \beta^{+}{ }_{2}=$ \\
$1\}, \mathrm{j}=1, \ldots, 52105201$ & 0,460526005 & 0,184210402 & 0,276315603 \\
\hline
\end{tabular}

Таблица 6. Модельные значения элементов подматриц $Z^{+} \mid Z^{+}{ }_{2}$ из матрицы $Z^{+}{ }_{m n}=\left[Z^{+} \mid Z^{+}{ }_{2}\right]$, имеющих заданные значения компонент вектора коэффициентов регрессии $B^{+}=\left(B^{+}{ }_{1}, \ldots, B^{+}{ }_{3}\right)^{T}$

\begin{tabular}{|c|c|c|c|c|c|c|c|}
\hline & № & $\mathrm{Z}^{+}{ }_{1}$ & $\mathrm{z}_{2}^{+}$ & $\mathrm{z}_{3}^{+}$ & $\mathrm{Z}^{+}$ & $\mathrm{z}^{+}{ }_{5}$ & $\mathrm{Z}_{6}^{+}$ \\
\hline 1 & 2 & 3 & 4 & 5 & 6 & 7 & 8 \\
\hline ROW & 1 & $\begin{array}{l}-7,319900 \mathrm{E}- \\
01\end{array}$ & $\begin{array}{l}-4,857400 \mathrm{E}- \\
01\end{array}$ & $\begin{array}{l}-4,668500 \mathrm{E}- \\
01\end{array}$ & $2,192300 \mathrm{E}+00$ & $2,031700 \mathrm{E}-01$ & $-0,6893844$ \\
\hline ROW & 2 & $\begin{array}{l}-1,962300 \mathrm{E}- \\
01\end{array}$ & $6,876200 \mathrm{E}-01$ & $6,970900 \mathrm{E}-02$ & $8,142700 \mathrm{E}-01$ & $7,612700 \mathrm{E}-02$ & $\begin{array}{l}0,29193986 \\
8\end{array}$ \\
\hline ROW & 3 & $\begin{array}{l}1,479400 \mathrm{E}- \\
01\end{array}$ & $\begin{array}{l}-9,498400 \mathrm{E}- \\
02\end{array}$ & $7,043700 \mathrm{E}-02$ & $\overline{1,913600 \mathrm{E}+00}$ & $\begin{array}{l}- \\
1,526000 \mathrm{E}+0 \\
0\end{array}$ & $-0,426588$ \\
\hline ROW & 4 & $\begin{array}{l}1,464000 \mathrm{E}+ \\
00\end{array}$ & $\begin{array}{l}-7,894800 \mathrm{E}- \\
01\end{array}$ & $\begin{array}{l}-6,551100 \mathrm{E}- \\
02\end{array}$ & $\begin{array}{l}-4,476700 \mathrm{E}- \\
01\end{array}$ & $9,825000 \mathrm{E}-01$ & 0,80305249 \\
\hline ROW & 5 & $\begin{array}{l}2,455400 \mathrm{E}+ \\
00\end{array}$ & $1,515600 \mathrm{E}+00$ & $1,409900 \mathrm{E}+00$ & $7,070400 \mathrm{E}-01$ & $\begin{array}{l}1,722900 \mathrm{E}+0 \\
0\end{array}$ & $\begin{array}{l}2,85401780 \\
8\end{array}$ \\
\hline ROW & 6 & $\begin{array}{l}- \\
1,099300 \mathrm{E}+ \\
00\end{array}$ & $2,345900 \mathrm{E}-01$ & $\begin{array}{l}-6,229100 \mathrm{E}- \\
01\end{array}$ & $\begin{array}{l}-4,457200 \mathrm{E}- \\
01\end{array}$ & $4,128900 \mathrm{E}-02$ & $\overline{0}-44476994$ \\
\hline ROW & 7 & $\begin{array}{l}6,926500 \mathrm{E}- \\
01\end{array}$ & $\begin{array}{l}-9,662700 \mathrm{E}- \\
01\end{array}$ & $\begin{array}{l}-5,374600 \mathrm{E}- \\
01\end{array}$ & $\begin{array}{l}- \\
1,443700 \mathrm{E}+00\end{array}$ & $\begin{array}{l}-1,929000 \mathrm{E}- \\
02\end{array}$ & $-\overline{0}-03559774$ \\
\hline ROW & 8 & $\begin{array}{l}2,164100 \mathrm{E}- \\
01\end{array}$ & $6,279000 \mathrm{E}-02$ & $3,316300 \mathrm{E}-01$ & $\begin{array}{l}-3,392200 \mathrm{E}- \\
01\end{array}$ & $2,810700 \mathrm{E}-01$ & $\begin{array}{l}0,22780471 \\
4\end{array}$ \\
\hline ROW & 9 & $\begin{array}{l}1,444600 \mathrm{E}+ \\
00\end{array}$ & $1,186600 \mathrm{E}+00$ & $1,798200 \mathrm{E}+00$ & $1,037800 \mathrm{E}+00$ & $9,131400 \mathrm{E}-01$ & $\begin{array}{l}1,66193703 \\
5\end{array}$ \\
\hline ROW & 10 & $\begin{array}{l}-2,397500 \mathrm{E}- \\
01\end{array}$ & $1,134000 \mathrm{E}+00$ & $8,509000 \mathrm{E}-01$ & $\begin{array}{l}-7,057100 \mathrm{E}- \\
01\end{array}$ & $\begin{array}{l}-4,179600 \mathrm{E}- \\
01\end{array}$ & $\begin{array}{l}0,29377322 \\
3\end{array}$ \\
\hline ROW & 11 & $\begin{array}{l}1,144900 \mathrm{E}+ \\
00\end{array}$ & $\begin{array}{l}-1,644500 \mathrm{E}- \\
01\end{array}$ & $\begin{array}{l}-6,312900 \mathrm{E}- \\
02\end{array}$ & $\begin{array}{l}-2,158000 \mathrm{E}- \\
01\end{array}$ & $\begin{array}{l}- \\
1,357400 \mathrm{E}+0 \\
0\end{array}$ & 0,2096692 \\
\hline ROW & 12 & $\begin{array}{l}1,042000 \mathrm{E}+ \\
00\end{array}$ & $\overline{1,512900 \mathrm{E}+00}$ & $\begin{array}{l}- \\
1,7978 \\
\end{array}$ & $\begin{array}{l}-8,986500 \mathrm{E}- \\
01\end{array}$ & 100E-01 & 1,03912167 \\
\hline ROW & 13 & $\begin{array}{l}-9,210600 \mathrm{E}- \\
01\end{array}$ & 2,325600E+00 & $2,337100 \mathrm{E}+00$ & $-\overline{1,259100 \mathrm{E}+00}$ & $9,887000 \mathrm{E}-01$ & 1,31416714 \\
\hline ROW & 14 & $\begin{array}{l}- \\
1,205000 \mathrm{E}+ \\
00\end{array}$ & $\begin{array}{l}-1,094000 \mathrm{E}- \\
01\end{array}$ & $5,297900 \mathrm{E}-02$ & $\begin{array}{l}-6,887800 \mathrm{E}- \\
01\end{array}$ & $\begin{array}{l}-7,782900 \mathrm{E}- \\
01\end{array}$ & $\begin{array}{l}- \\
1,09395696 \\
\end{array}$ \\
\hline ROW & 15 & $\begin{array}{l}-6,065900 \mathrm{E}- \\
01\end{array}$ & $\begin{array}{l}-2,017600 \mathrm{E}- \\
01\end{array}$ & $2,382100 \mathrm{E}-01$ & $7,019500 \mathrm{E}-01$ & $\begin{array}{l}-4,405800 \mathrm{E}- \\
01\end{array}$ & 0,71652596 \\
\hline ROW & 16 & $\begin{array}{l}-4,529500 \mathrm{E}- \\
01\end{array}$ & $7,584200 \mathrm{E}-01$ & $5,990200 \mathrm{E}-01$ & 7,692700E-01 & $\begin{array}{l}- \\
1,564200 \mathrm{E}+0 \\
0\end{array}$ & $\begin{array}{l}- \\
0,44969978\end{array}$ \\
\hline ROW & 17 & $\begin{array}{l}2,798000 \mathrm{E}- \\
01\end{array}$ & $\begin{array}{l}-9,469800 \mathrm{E}- \\
01\end{array}$ & $\begin{array}{l}-6,650600 \mathrm{E}- \\
01\end{array}$ & $7,601100 \mathrm{E}-01$ & $\begin{array}{l}1,196400 \mathrm{E}+0 \\
0\end{array}$ & $\begin{array}{l}- \\
0,71187649\end{array}$ \\
\hline ROW & 18 & $\begin{array}{l}1,397900 \mathrm{E}+ \\
00\end{array}$ & $\begin{array}{l}-4,873200 \mathrm{E}- \\
01\end{array}$ & $\begin{array}{l}- \\
1,047000 \mathrm{E}+00\end{array}$ & $\begin{array}{l}-1,181400 \mathrm{E}- \\
01\end{array}$ & $3,582300 \mathrm{E}-01$ & $-0,9225874$ \\
\hline
\end{tabular}




\begin{tabular}{|c|c|c|c|c|c|c|}
\hline \multirow{4}{*}{ Impact Factor: } & ISRA (India) & $=3.117$ & SIS (USA) & $=0.912$ & ICV (Poland) & $=6.630$ \\
\hline & ISI (Dubai, UAI & $=0.829$ & РИНЦ (Russia & $=0.156$ & PIF (India) & $=1.940$ \\
\hline & GIF (Australia) & $=0.564$ & ESJI (KZ) & $=8.716$ & IBI (India) & $=4.260$ \\
\hline & JIF & $=1.500$ & SJIF (Morocce & $=5.667$ & OAJI (USA) & $=0.350$ \\
\hline
\end{tabular}

\begin{tabular}{|c|c|c|c|c|c|c|c|}
\hline ROW & 19 & $\begin{array}{l}1,794900 \mathrm{E}- \\
01\end{array}$ & $1,411900 \mathrm{E}+00$ & $1,079900 \mathrm{E}+00$ & $2,513400 \mathrm{E}-01$ & $\begin{array}{l}-9,364700 \mathrm{E}- \\
01\end{array}$ & 0,50746346 \\
\hline ROW & 20 & $\begin{array}{l}-1,325700 \mathrm{E}- \\
01\end{array}$ & $1,093500 \mathrm{E}+00$ & $1,102000 \mathrm{E}+00$ & $1,242000 \mathrm{E}+00$ & $\begin{array}{l}1,851800 \mathrm{E}+0 \\
0\end{array}$ & $\begin{array}{l}0,99463853 \\
8\end{array}$ \\
\hline average & & 0,00000 & 0,00000 & 0,00000 & 0,00000 & 0,00000 & 0,00000 \\
\hline $\begin{array}{l}\text { st } \\
\text { deviati } \\
\text { on }\end{array}$ & & 1,00000 & 1,00000 & 1,00000 & 1,00000 & 1,00000 & 1,00000 \\
\hline
\end{tabular}

Извлечение знаний проведем аналогично изложен ному в статье [2].

Поменяем значения выделенных элементов из матрицы собственных векторов $\mathbf{C}^{+} \mathbf{1 1}$ (Таблица 1) на назначенные экспертом значения. Реализуем программу-таблицу (Таблица 1, Рисунки 1,2). Элементы из матрицы собственных векторов $\mathbf{C}^{+}{ }_{11}$ изменились, а значения выделенных элементов не изменились: они являются математически введенными индикаторами наличия знаний. Соответствующая ей матрица собственных чисел $\Lambda^{+}{ }_{11}=\operatorname{diag}(1.8978,1.05373,1.05373,0.86461$,

0.13012) также является результатом математического решения Оптимизационной Задачи и предварительного привнесения (добавления) знаний в $\mathrm{C}^{+} 11$ путем внесения заметных значений (Таблица 1). Покажем как эти значения когнитивно моделируют смыслы. Сперва были [2] назначены доли информации для 4-х типов валидных показателей $\mathrm{y}_{1}, \mathrm{y}_{2} \quad \mathrm{y}_{3}, \mathrm{y}_{4}$ «глубинного» морального сознания. Будем считать их соответствующих «смыслам» (направлениям деятельности) 4-х ниже описанных департаментов. Для каждого из них мы смоделировали 2,5,1, $3 \mathrm{z}^{+}$-переменных из их

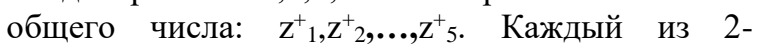
членных,5-членных, 1- членных, 3-членных отделов (subdivisions) имеет свой валидный «смысл», равный сумме смыслов $\mathrm{z}^{+}$-переменных с значимыми смыслами весомых значений компонентов соответствующего собственного вектора.

Наши $\mathrm{z}^{+}$-переменные удовлетворяют соотношениям ПМ МЛРА, ОМ МЛРА и ОМ ГК, при этом $5 \mathrm{z}^{+}$-переменные функционально зависят от одной $\mathrm{z}^{+}$-переменной $\mathrm{z}_{6}^{+}$, смысл $\mathrm{z}_{6}^{+}$когнитивно определен в [16] и сформулирован так: смысл $\left(\mathrm{z}^{+}{ }_{6}\right)=$ «стремится обмануть, а не добросовестно сделать что-либо». Ссмысл $\left(\mathrm{z}^{+}{ }_{6}\right)=$ «стремится обмануть, а не добросовестно сделать что-либо» когнитивно сформулирован 1 раз как смысл $\mathrm{z}^{+}$-переменной, являющегося частью смысла валидной $\mathrm{y}$ переменной у Зависимость смысла $\mathrm{z}_{6}^{+}$от смысла у -переменной $\mathrm{y}_{3}$ обозначено так: смысл $\left(\mathrm{y}_{3}, \mathrm{Z}_{6}^{+}\right)=$«стремится обмануть, а не добросовестно сделать что-либо». Смыслы новых $\mathrm{z}^{+}$-переменных (из ОМ МЛРА) мы приравняли к смыслам прежних z-переменных (из ОМ ГК). Но в ОМ МЛРА $\mathrm{z}^{+}$-переменные подчинены уравнению регрессии $\mathrm{Z}^{+}{ }_{6}=\beta^{+}{ }_{1} \mathrm{Z}^{+}{ }_{1}+\beta^{+}{ }_{2} \mathrm{Z}^{+}{ }_{2}+\ldots+\beta^{+}{ }_{5} \mathrm{Z}^{+}{ }_{5}$ и смысл $\mathrm{Z}^{+}-$ переменной $\mathrm{z}_{6}^{+}$равен сумме смыслов не всех $5 \mathrm{z}^{+}$переменных. Смысл $\mathrm{z}^{+}$-переменной $\mathrm{z}^{+}$зависит от числа значимых коэффициентов регресс сии. Такими коэффициентами являются $3\left(\beta_{3}=5\right.$, $\left.\beta_{1}=4, \beta_{5}=3\right)$ из 5-ти $\left(\beta_{1}=4, \beta_{2}=1, \beta_{3}=5, \beta_{4}=2, \beta_{5}=3\right)$ назначенных нами коэффициентов регрессии.

Новые добавляемые смыслы обозначим как Смысл $\left(z_{6}^{+} ; \beta_{3}, \beta_{5}\right)$, Смысл $\left(z_{6}^{+} ; \beta_{1}=4, \beta_{2}=4, \beta_{3}=3, \beta_{4}=2, \beta\right.$ $\left.{ }_{5}=3\right)$, Смысл $\left(\mathrm{z}_{6}^{+} ; \beta_{1}\right)$, Смысл $\left(\mathrm{z}_{6}^{+} ; \beta_{2}=1, \beta_{3}=5, \quad \beta_{4}=2\right.$, $\left.\beta_{5}=3\right)$.

Перейдем к этапу когнитивного извлечения знаний. Когнитивный смысл передаем фразой из 3 слов. Кортеж $\left(\beta_{1}=5, \beta_{2}=4, \beta_{3}=3\right)$ с тремя смыслами (казнить нельзя помиловать) озвучивается фразой «помилования $\left(\beta_{3}=3\right)$ не добившиеся $\left(\beta_{2}=4\right)$ казненные $\left(\beta_{1}=5\right)$ ». Кортеж $\left(\beta_{1}=5, \beta_{2}=4, \beta_{3}=3\right)$ с тремя смыслами (помиловать нельзя казнить) озвучивается фразой «казни $\left(\beta_{3}=3\right)$ избежавшие $\left(\beta_{2}=4\right)$ помилованные $\left(\beta_{1}=5\right) »$. Перед существительным стоят 2 прилагательные с возрастающей силами $\left(\beta_{5}=3\right.$ и $\left.\beta_{1}=4\right)$.

Такой «стандарт» свободен от разночтений, стилистически несовершенен, но упрощает когнитивный выбор смыслов и уменьшает степень субъективизма, присущего когнитивному моделированию [18], впервые примененный формализации описаний ситуаций в политологии с многосмысловыми фразами, даже противоречащие общепринятой фразе.

В применяемой в этой статье «грамматике» фразы не являются выбором регулярных выражений для когнитивных смыслов. Они не аналогичны по возможностям использующимся некоторыми текстовыми редакторами и утилитами способу поиска и подстановки текста. В способ поиска и подстановки текста «при помощи регулярных выражений можно задать шаблоны, позволяющие: найти все последовательности символов «кот» в любом контексте, как то: «кот», «котлета», «терракотовый»; найти отдельно стоящее слово «кот» и заменить его на «кошка»; найти слово «кот», которому предшествует слово «персидский» или «чеширский»; убрать из текста 


\begin{tabular}{|c|c|c|c|c|c|c|}
\hline \multirow{4}{*}{ Impact Factor: } & ISRA (India) & $=3.117$ & SIS (USA) & $=0.912$ & ICV (Poland) & $=6.630$ \\
\hline & ISI (Dubai, UAI & $=0.829$ & РИНЦ (Russia & $=0.156$ & PIF (India) & $=1.940$ \\
\hline & GIF (Australia) & $=0.564$ & ESJI (KZ) & $=8.716$ & IBI (India) & $=4.260$ \\
\hline & JIF & $=1.500$ & SJIF (Morocce & $=5.667$ & OAJI (USA) & $=0.350$ \\
\hline
\end{tabular}

все предложения, в которых упоминается слово кот или кошка» ${ }^{5}$. Регулярные грамматики (в нашем случае - праволинейная грамматика) являются подмножеством контекстно-свободных. Такой «стандарт» не связан с типами грамматик контекстно-свободный или контекстнозависимый ${ }^{6}$

Когнитивная модель структуры муниципального органа по мониторингу моральной среды для подвидов человеческих ресурсов

Когнитивная модель структуры муниципальных органов по мониторингу моральной среды для видов человеческих ресурсов состоит из 4-х валидных моральных показателей, из 12 показателей, соответствующих своему валидному моральному показателю, из 6 показателей, обладающих суммарным смыслом той или иной части из вышеназванных смыслов 12 показателей.

Ниже показаны обоснования для учреждения 4-х Департаментов мониторинга моральной среды у человеческих ресурсов, в состав которых целесообразно включить 12 узких специалистов, объединенных в 6 отделов. Число специалистов одного вида (из 12) должно быть кратно фактической нагрузке. Вопросы нормирования труда каждого специалистов и вопрос оплаты их работы мы не рассматриваем.

Дополнительных знаний, в присутствии которых мы уверены, зная «коэффициенты регрессии, содержащие... когнитивно извлекаемые знания» [1], существует 6 - по одному на каждое собственное число $\lambda_{1}, \lambda_{2}, \lambda_{3}, \lambda_{4}$, $\lambda_{5}, \lambda_{6}$. Их смыслы выражают смыслы регрессионных коэффициентов в линейных комбинациях $\mathrm{m}$ значений $\mathrm{z}^{+}$-переменной $\mathrm{z}^{+}{ }_{6}=\beta^{+}{ }_{1} \mathrm{Z}^{+}{ }_{1}+\beta^{+}{ }_{2} \mathrm{z}^{+}{ }_{2}+\ldots+\beta^{+}{ }_{5} \mathrm{Z}^{+}$. Смыслы $\beta^{+} \mathrm{Z}^{+}{ }_{1}, \beta^{+}{ }_{2} \mathrm{Z}^{+}{ }_{2}, \ldots, \beta^{+} \mathrm{Z}^{+}{ }_{5}$ отличаются от смыслов линейных комбинаций $\mathrm{c}$ «весами» $\mathrm{c}_{1 \mathrm{j}}, \mathrm{c}_{2 \mathrm{j}} \ldots \mathrm{c}_{\mathrm{nj}}$ (компонентами ј-го собственного вектора) при каждой из $\mathrm{n} \mathrm{z}^{+}$-переменных. «Веса» подчиняются как равенству $\mathrm{c}^{2}{ }_{1 \mathrm{j}}+\mathrm{c}^{2}{ }_{2 \mathrm{j}} \ldots+\mathrm{c}^{2}{ }_{\mathrm{nj}}=1$, так и равенству $\mathrm{c}^{2}{ }_{\mathrm{i} 1}+\mathrm{c}^{2}{ }_{\mathrm{i} 2} \ldots+\mathrm{c}^{2}{ }_{\mathrm{in}}=1$. Эти «веса» определяют другую систему у-переменных, имеющих смыслы валидных переменных [6] с известными смыслами, влияющими на все смыслы других вышеназванных переменных и смыслы разных сумм коэффициентов регрессии.

К ранее извлеченным знаниям по модели ОМ ГК добавим новые знания (смыслы), извлеченные при помощи ОМ МЛРА. Новые смыслы

${ }^{5}$ www.wikipedia.org/wiki/Регулярные выражения ${ }^{6}$ Хопкрофт Д., Мотвани Р., Ульман Д. Введение в теорию автоматов, языков и вычислений, 2-е изд. когнитивно извлекаем из регрессионных уравнений, содержащих разные совокупности коэффициентов регрессии. Они таковы, что их смыслы когнитивно равны смыслу Смысл $\left(\mathrm{z}_{6}^{+}\right.$; «влияющие коэффициентов регрессии» $)=$ «стремится обмануть, а не добросовестно сделать что-либо», где вместо слов «влияющие коэффициентов регрессии» дан перечень коэффициентов регрессии, присутствующих в регрессионном уравнении для зависимой переменной $\mathrm{Z}^{+}$, используемого при когнитивном определении суммарного смысла Смысл( $\mathrm{z}_{6}^{+}$;«влияющие коэффициентов регрессии» $)=$ «стремится обмануть, а не добросовестно сделать что-либо» является левой частью формируется как элемент левой части праволинейной грамматики, а правя часть из суммы фраз учитывающих значение каждого коэффициента корреляции.

Смысл $\left(\mathrm{z}_{6}^{+} ; \beta_{3}, \beta_{5}\right)$ когнитивно определяем так. В валидной переменной у1 (ее вес равен $37.96 \%$, $\left.\lambda_{1}=1.8978\right)$ в правую часть нами вложены в 2 компоненты 1-го собственного вектора 2 весомых значений: $\operatorname{corr}\left(\mathrm{y}_{1}, \mathrm{z}_{5}\right)=\mathrm{c}_{51}=0,5, \quad \operatorname{corr}\left(\mathrm{y}_{1}, \mathrm{z}_{3}\right)=\mathrm{c}_{31}=0,5$. Наличие валидной переменной $\mathbf{y}_{1}$ (из-за наличия математического равенства $\mathrm{y}^{+}{ }_{\mathrm{ij}}=\mathrm{Z}^{+}{ }_{\mathrm{i} 1} \mathrm{c}^{+}{ }_{1 \mathrm{j}}+\ldots+\mathrm{z}^{+}{ }_{\mathrm{i} 5} \mathrm{c}^{+}{ }_{5 \mathrm{j}}$, $\left.\mathrm{j}=1, \ldots, 5, \mathrm{i}=1, \ldots, 20, \quad \mathrm{z}^{+}{ }_{6}=\beta^{+}{ }_{1} \mathrm{Z}^{+}{ }_{1}+\beta^{+}{ }_{2} \mathrm{Z}^{+}{ }_{2}+\ldots+\beta^{+}{ }_{5} \mathrm{Z}^{+}{ }_{5}\right)$ когнитивно моделирует смысль $\mathrm{Z}-$ переменным $\mathrm{z}^{+}{ }_{3}, \mathrm{Z}^{+}$. $\left(\mathrm{y}_{1}^{+}, \mathrm{z}^{+}{ }_{3}\right)=$ «порядочность» [2]. Смысл $\mathrm{z}^{+}-$ переменной $\mathrm{z}_{6}^{+}$зависит от числа значимых коэффициентов регрессии. Такими коэффициентами являются $2\left(\beta_{3}=5, \beta_{5}=3\right)$ из 3 -х значимых $\left(\beta_{1}=4, \beta_{3}=5, \beta_{5}=3\right)$. Всего их 5: $\left(\beta_{1}=4, \beta_{2}=1\right.$, $\left.\beta_{3}=5, \beta_{4}=2, \beta_{5}=3\right)$.

Наличие валидной переменной $\mathrm{y}^{+}$ когнитивно моделирует смыслы $\mathrm{z}^{+}$-переменным $\mathrm{z}^{+}{ }_{1}, \mathrm{z}^{+}{ }_{2}, \ldots, \mathrm{z}^{+}$. Эти смыслы не меняются, меняются лишь степени выраженности смыслов. Измеренные нами силы смыслов должны изменяться как по времени, так и по местам их измерения. Следующие $5 \mathrm{z} \mathrm{z}^{+}$-переменных $\mathrm{z}^{+}{ }_{1}, \mathrm{Z}^{+}{ }_{2}, \ldots, \mathrm{z}^{+}{ }_{5}$ функционально связаны с $\mathrm{y}^{+}-$ переменной $\mathrm{y}_{2}^{+}$(из-за наличия математического равенства $\quad \mathrm{y}^{+}{ }_{\mathrm{ij}}=\mathrm{z}^{+}{ }_{\mathrm{i} 1} \mathrm{c}^{+}{ }_{1 \mathrm{j}}+\ldots+\mathrm{z}^{+}{ }_{\mathrm{i} 5} \mathrm{c}^{+}{ }_{5 \mathrm{j}}, \quad \mathrm{j}=1, \ldots, 5$, $\mathrm{i}=1, \ldots, 20)$ и имеют когнитивные смыслы: смысл $\left(\mathrm{y}^{+}{ }_{2}, \mathrm{z}^{+}{ }_{1}\right)=$ «лицо низкой социальной активности», смысл $\left(\mathrm{y}^{+}, \mathrm{z}_{2}^{+}\right)=$«низкая зарплата», смысл $\left(\mathrm{y}_{2}^{+}, \mathrm{z}_{3}^{+}\right)=$«порядочность», смысл $\left(\mathrm{y}^{+}{ }_{2}, \mathrm{z}_{4}^{+}\right)=$ «проявление деспотизма», смысл $\left(\mathrm{y}^{+}{ }_{2}, \mathrm{Z}^{+}\right)=$ «честность».

Наличие валидной переменной $\mathrm{y}^{+}$ когнитивно моделирует смысл $\mathrm{z}^{+}$-переменной $\mathrm{z}^{+}$.

:Пер. с англ.-Москва, Издательский дом «Вильямс», 2002. -528 с. 


\begin{tabular}{|c|c|c|c|c|c|c|}
\hline \multirow{4}{*}{ Impact Factor: } & ISRA (India) & $=3.117$ & SIS (USA) & $=0.912$ & ICV (Poland) & $=6.630$ \\
\hline & ISI (Dubai, UAI & $=0.829$ & РИНЦ (Russia & $=0.156$ & PIF (India) & $=1.940$ \\
\hline & GIF (Australia) & $=0.564$ & ESJI (KZ) & $=8.716$ & IBI (India) & $=4.260$ \\
\hline & JIF & $=1.500$ & SJIF (Morocce & $=5.667$ & OAJI (USA) & $=0.350$ \\
\hline
\end{tabular}

ее смысл $\left(\mathrm{y}^{+}{ }_{4}, \mathrm{z}^{+}{ }_{5}\right)=$ античестность, а величину его выраженности мы назначили равной 0.5: $\mathrm{c}_{25}=0,5000$.

Все математически введенные (субъективно назначенные) значения весомых индикаторов присутствия знаний (смыслов $\mathrm{z}$-переменных $\mathrm{z}^{+} 1, \mathrm{Z}^{+}{ }_{2}, \ldots, \mathrm{Z}^{+}$) выступают в виде точных равенств из ООЗ МЛРА. В соответствии с математической моделью ООМ МЛРА и этапа назначения желаемого набора коэффициентов регрессии $\beta^{+}{ }_{1}, \ldots, \beta^{+}{ }_{5}$ мы приступим к обратному процессу к этапу когнитивного извлечения введенных ранее знаний. Извлечение знаний проведем аналогично изложенному в статье [2].

Так как смысл $\left(\mathrm{y}^{+}{ }_{1}, \mathrm{z}^{+}\right)=$«античестность», смысл $\left(\mathrm{y}^{+}{ }_{1}, \mathrm{Z}^{+}{ }_{3}\right)=$ порядочность», то Смысл $\left(\mathrm{z}^{+}{ }_{6}\right.$ $\left., \beta_{3}, \beta_{5}\right)=$ честная порядочность (в отличие от демонстративной порядочности). Этот смысл извлекаем из соотношения: смысл $\left(\beta_{1} \mathrm{z}^{+}{ }_{1}+\beta_{2} \mathrm{Z}^{+}{ }_{2}+\ldots+\beta_{5} \mathrm{Z}^{+}{ }_{5}\right)=$ смысл $\left(\beta_{1} \mathrm{z}^{+}\right)+$смысл $\left.\left(\beta_{2} Z^{+}\right)\right)+$смысл $\left(\beta_{3} Z^{+}{ }_{3}\right)+$ смысл $\left(\beta_{4} Z^{+}{ }_{4}\right)+$ смысл $\left(\beta_{5} Z^{+}{ }_{5}\right)$.

Смысл $\left(z_{6}^{+} ; \beta_{3}, \beta_{5}\right)$ когнитивно определяем так. В левой части смыслового равенства имеем смысл $\left(\mathrm{y}_{3}, \mathrm{z}_{6}\right)=$ «стремится обмануть, а не добросовестно сделать что-либо». В правой части смыслового равенства имеем смысл, равный линейной комбинации 2-х смыслов Смысл $\left(\mathrm{z}^{+}{ }_{6}, ; \beta^{+}{ }_{1}, \ldots, \beta^{+}{ }_{5}\right)=$ Смысл $\left(\mathrm{z}^{+}{ }_{6} ; \beta_{1}, \ldots, \beta_{5}\right)$.

Ниже будем применять удобное смысловое равенство, зависящее от коэффициентов регрессии $\quad \beta_{1}, \ldots, \beta_{5} . \quad$ Смысл $\left(\mathrm{z}^{+}{ }_{6} ; \beta_{3}, \beta^{+}{ }_{5}\right)=$ $5 *$ [«антипорядочность»]+3*[«античестность»] $=$ нечестная преступная анти порядочность. Заметим, что честная порядочность отличается от демонстративной порядочности.

Для валидной переменной $\mathrm{y}^{+}{ }_{1} \quad 37,96 \%$ $\left(\lambda^{+}=1.8978\right) \quad$ извлеченных знаний отражено в смысле порядочность». В других извлекаемых нами знаниях отражаются негативные смыслы. Их объемы равны $21.07 \%, 21.07 \%, 17.29 \%, 2.60 \%$.

Для валидной переменной $\mathrm{y}^{+}{ }_{2}$ зависимая $\mathrm{z}^{+}-$ переменная $\mathrm{Z}_{6}^{+}{ }_{6}$ в правой части смыслового равенства имеет смысл, равный сумме 5 смыслов. Это обусловлено наличием математического равенства $\mathrm{y}^{+}{ }_{\mathrm{ij}}=\mathrm{Z}^{+}{ }_{\mathrm{i} 1} \mathrm{c}^{+}{ }_{1 \mathrm{j}}+\ldots+\mathrm{z}^{+}{ }_{\mathrm{i} 5} \mathrm{c}^{+}{ }_{5 \mathrm{j}}, \quad \mathrm{j}=1, \ldots, 5$, $\mathrm{i}=1, \ldots, 20$. Формируем фразу для правой части смыслового равенства.

Официальные названия департаментов обычно соответствуют концепции внутренней политики, мы даем лишь функциональные смыслы их направлениям работы.

В валидной переменной $\mathrm{y}^{+}{ }_{1}$ (ее вес равен $\left.37,96 \%, \lambda_{1}=1.8978\right)$ нами вложены в 2 компоненты 1-го собственного вектора 2 весомых значения: $\operatorname{corr}\left(\mathrm{y}_{1}, \mathrm{z}_{5}\right)=\mathrm{c}_{51}=0,5$, $\operatorname{corr}\left(\mathrm{y}_{1}, \mathrm{z}_{3}\right)=\mathrm{c}_{31}=0,5$.

Смысл $\left(\mathrm{y}_{1}, \mathrm{z}_{5}\right)=$ «античестность», смысл $\left(\mathrm{y}_{1}, \mathrm{z}_{3}\right)=\langle$ «порядочность».
Наличие валидной переменной $\mathrm{y}^{+}{ }_{1}$ (из-за математического равенства $\mathrm{y}^{+}{ }_{\mathrm{ij}}=\mathrm{z}^{+}{ }_{\mathrm{i} 1} \mathrm{c}^{+}{ }_{1 \mathrm{j}}+\ldots+\mathrm{z}^{+}{ }_{\mathrm{i} 5 \mathrm{c}^{+}}{ }_{5 \mathrm{j}}$, $\mathrm{j}=1, \ldots, 5, \mathrm{i}=1, \ldots, 20)$ когнитивно моделирует cмыслы $\mathrm{Z}^{+}$-переменным $\mathrm{z}_{3}^{+}, \mathrm{z}_{5}^{+}$. Смысл $\left(\mathrm{y}_{1}, \mathrm{z}_{5}\right)=$ «античестность», смысл $\left(\mathrm{y}_{1}, \mathrm{z}_{3}\right)=$ «порядочность» [2], а смысл $\mathrm{z}^{+}$-переменной $\mathrm{z}_{6}^{+}$зависит от значимых коэффициентов регрессии. Такими коэффициентами являются $2: \beta_{3}=5, \beta_{5}=3$ (из $3-\mathrm{x}$ значимых $\beta_{1}=4, \beta_{3}=5, \beta_{5}=3$. Всего их 5: $\left(\beta_{1}=4, \beta_{2}=1\right.$, $\beta_{3}=5, \beta_{4}=2, \beta_{5}=3$ ).

Для валидной переменной $\mathrm{y}^{+}{ }_{1}$ зависимая $\mathrm{z}^{+}-$ переменная $\mathrm{z}_{6}^{+}$имеет еще дополнительные смыслы, равные сумме 2-х смыслов Смысл $\left(\mathrm{z}_{6}^{+} ; \beta_{3}=5, \beta_{5}=3\right)=\left[0.426060505^{*}\right.$

порядочность $]+\left[\right.$ смысл $\left(\mathrm{y}_{1}, \mathrm{z}_{5}\right)=0.276315603 *$

«античестность»]=античестная порядочность, отличающаяся, например, от демонстративной порядочности.

Для валидной переменной $\mathrm{y}^{+}{ }_{2}$ (ее вес равен $\left.34.59 \%, \lambda_{2}=1,383727\right)$ зависимая $\mathrm{z}^{+}-$переменная $\mathrm{z}_{6}^{+}$ имеет смысл, равный сумме 5 смыслов. Смысл $\left(\mathrm{z}_{6}^{+} ; \beta_{1}=4, \beta_{2}=1, \beta_{3}=5, \beta_{4}=2, \beta_{5}=3\right)=$ $0.364208048 *$ смысл $\left(\mathrm{z}^{+}{ }_{1}\right)+$

$0.092105201 *$ смысл $\left(\mathrm{z}^{+}\right)+0.426060505 *$ смысл $\left(\mathrm{z}^{+}{ }_{3}\right)$ $+0.184210402 *$ смысл $\left(\mathrm{z}^{+}{ }_{4}\right)+0.276315603 *$ смысл $\left(\mathrm{z}^{+}{ }_{5}\right.$ )$=4 *[$ «лицо низкой социальной активности»] +1 *[«низкая зарплата»] $+5 *$ [«антипорядочность»] $+2 *[$ «проявление

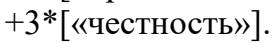

При этом нам легче опираться на значения экспертных значений коэффициентов регрессии $\beta_{1}=4, \beta_{2}=1, \beta_{3}=5, \beta_{4}=2, \beta_{5}=3$. Тогда Смысл $\left(\mathrm{z}^{+}{ }_{6} ; \beta_{1}=4, \beta_{2}=1, \beta_{3}=5, \beta_{4}=2, \beta_{5}=3\right)=4 *$ [«лицо низкой социальной активности»]+1*[«низкая зарплата»] $+5 *[$ «антипорядочность»] $+2[$ «проявлен ие деспотизма»] $+3 *[$ «естность»] $=$ нечестная рабская антипорядочность.

Для валидной переменной $\mathrm{y}_{3}^{+}$(ее вес равен $\left.34.157 \%, \lambda_{3}=1,366273\right)$ зависимая $\mathrm{Z}^{+}$-переменная $\mathrm{Z}_{6}^{+}$дополнительно имеет смысл, выражаемый фразой «батрак»: Смысл $\left(\mathrm{z}_{6}^{+} ; \beta_{1}=4\right)=4 *[$ «лицо низкой социальной активности»]=батрак. Эта фраза выделяет то, что обладание которым индивиду присуще не своей воле. Смысл фразы «батрак» отличается от смысла слова «рабская», являющейся «генетической» характеристикой индивида. При этом используем математическую модель главных компонент [1]. Так как $\mathrm{z}^{+}-$ переменная $\mathrm{z}_{6}^{+}$имеет когнитивный смысл: Ссмысл $(\mathrm{y} 3, \mathrm{z6})=$ «стремится обмануть, а не добросовестно сделать что-либо», то в рамках другой математической модели - ОМ МЛРА, применяемой в данной статье, смысл $\mathrm{z}^{+}-$ переменной $\mathrm{z}^{+} 6$ дополняется смыслом одной (из 5ти) $\mathrm{z}^{+}$-переменной (из-за наличия математического равенства $\mathrm{z}_{6}^{+}=\beta_{1} \mathrm{Z}^{+}{ }_{1}+$ $\beta_{2} \mathrm{Z}_{2}{ }_{2}+\ldots+\beta_{5} \mathrm{Z}_{5}^{+}$) передаваемой фразой «батрак». 


\begin{tabular}{|c|c|c|c|c|c|c|}
\hline \multirow{4}{*}{ Impact Factor: } & ISRA (India) & $=3.117$ & SIS (USA) & $=0.912$ & ICV (Poland) & $=6.630$ \\
\hline & ISI (Dubai, UAI & $=0.829$ & РИНЦ (Russia & $=0.156$ & PIF (India) & $=1.940$ \\
\hline & GIF (Australia) & $=0.564$ & ESJI (KZ) & $=8.716$ & IBI (India) & $=4.260$ \\
\hline & JIF & $=1.500$ & SJIF (Morocce & $=5.667$ & OAJI (USA) & $=0.350$ \\
\hline
\end{tabular}

Смысл «стремится обмануть, а не добросовестно сделать что-либо» является скрытым, один из примеров недавно озвучен в СМИ Республики Казахстан: участились случаи фиктивных разводов в многодетных семьях, их скрытая цель - получение реальной денежной помощи, это случай обмана индивидом государства. На занятиях по дисциплине «Информационный бизнес» (тема «Приманка и крючок») студенты приводили мне примеры «обмана» для клиентов, побуждающие их к потреблению услуг в интересах бизнеса. Мы рассматриваем прежде всего обманы и другие моральные качества на ином индивидуальном уровне.

В валидной переменной $\mathrm{y}_{4}^{+}$(ее вес равен $\left.25 \%, \lambda_{4}=1.000\right)$ нами вложены 4 индикатора присутствия знаний (в 4 компоненты 4-го собственного вектора - 4 весомых значений: $\operatorname{corr}\left(\mathrm{y}_{4}, \mathrm{z}_{2}\right)=\mathrm{c}_{24}=0.5, \quad \operatorname{corr}\left(\mathrm{y}_{4}, \mathrm{z}_{3}\right)=\quad \mathrm{c}_{34}=0.5$, $\operatorname{corr}\left(\mathrm{y}_{4}, \mathrm{z}_{4}\right)=\mathrm{c}_{44}=-0.5, \operatorname{corr}\left(\mathrm{y}_{4}, \mathrm{z}_{5}\right)=\mathrm{c}_{54}=-0.5$. Наличие валидной переменной у4 (из-за математического

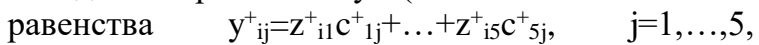
$\mathrm{i}=1, \ldots, 20)$ когнитивно моделирует смыслы $\mathrm{z}-$ переменным $\mathrm{z}_{2}, \mathrm{z}_{3}, \mathrm{z}_{4}, \mathrm{z}_{5}$ следующие смыслы: смысл $\left(\mathrm{y}_{4}, \mathrm{z}_{2}\right)=$ «низкая зарплата», смысл $\left(\mathrm{y}_{4}, \mathrm{z}_{3}\right)=$ =антипорядочность, смысл $\left(\mathrm{y}_{4}, \mathrm{z}_{4}\right)=$ «проявление деспотизма», смысл $\left(\mathrm{y}_{4}, \mathrm{z}_{5}\right)=$ античестность. Тогда смысл $\mathrm{z}^{+}$ переменной $\mathrm{Z}_{6}^{+}$зависит от смыслов значимых коэффициентов регрессии. Такими коэффициентами являются 2 коэффициента регрессии $\left(\beta_{3}=5, \beta_{5}=3\right)$. Для валидной переменной $\mathrm{y}_{4}$ зависимая $\mathrm{z}-$-переменная $\mathrm{z}_{6}^{+}$имеет смысл, равный линейной комбинации 2-х смыслов. Смысл $\left(\mathrm{z}_{6}^{+}, \beta_{3}=5, \beta_{5}=3\right)==5 *$ [«антипорядочность»]+

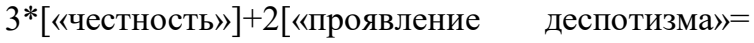
честная вынужденная антипорядочность= коррупция госслужащих.

Таблица 7. Значения имен, смыслов у- и z-переменных, коэффициентов (y,z)-корреляций

\begin{tabular}{|c|c|c|c|c|c|}
\hline \multicolumn{6}{|c|}{$\begin{array}{l}\text { Весовая и смысловая характеристика валидных и } \\
\text { модельных } z \text {-переменных в }\left(\beta, C^{+}{ }_{11}\right)-\text { выборке ООМ МЛРА }\end{array}$} \\
\hline 1 & 2 & 3 & 4 & & 5 \\
\hline $\begin{array}{l}\text { «Вес» } \\
\text { валидной } \\
\text { переменной }\end{array}$ & $\begin{array}{l}\text { Смысл } \\
\text { валидной } \\
\text { переменной }\end{array}$ & 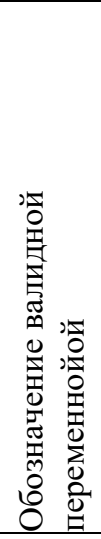 & $\begin{array}{l}\text { Значения «весов» } \mathrm{z-} \\
\text { пере менной, линейно } \\
\text { входя щих } \\
\text { валидную } \\
\text { переменную }\end{array}$ & 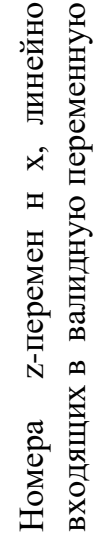 & $\begin{array}{l}\text { Смысли } \text { z-переменных, линейно } \\
\text { входящих в валидную переменную }\end{array}$ \\
\hline$\lambda_{1}=1,75$ & $\begin{array}{l}\text { «по правде } \\
\text { (правильно) } \\
\text { поступать» }\end{array}$ & $\mathrm{y}_{1}$ & $\begin{array}{l}\operatorname{corr}\left(\mathrm{y}_{1}, \mathrm{z}_{5}\right)=\mathrm{c}_{51} \\
\mathbf{0 , 5 1 9 1} \\
\operatorname{corr}\left(\mathrm{y}_{1}, \mathrm{z}_{3}\right)=\mathrm{c}_{31} \\
\mathbf{0 , 4 4 8 1}\end{array}$ & 3,5 & $\begin{array}{l}\text { смысл }\left(\mathrm{y}_{1}, \mathrm{z}_{5}\right)=\text { «античестность» } \\
\text { смысл }\left(\mathrm{y}_{1}, \mathrm{z}_{3}\right)=\text { порядочность }\end{array}$ \\
\hline$\lambda_{2}=1,383727$ & $\begin{array}{l}\text { «принудить } \\
\text { к...», }\end{array}$ & $\mathrm{y}_{2}$ & $\begin{array}{l}\operatorname{corr}\left(\mathrm{y}_{2}, \mathrm{z}_{1}\right)=\mathrm{c}_{21}=\mathbf{- \mathbf { 0 , 5 }} \\
\operatorname{corr}\left(\mathrm{y}_{2}, \mathrm{z}_{2}\right)=\mathrm{c}_{22}=\mathbf{- \mathbf { 0 , 4 }} \\
\operatorname{corr}\left(\mathrm{y}_{2}, \mathrm{z}_{3}\right)=\mathrm{c}_{32}=0,5 \\
\operatorname{corr}\left(\mathrm{y}_{2}, \mathrm{z}_{4}\right)=\mathrm{c}_{42}=\mathbf{- 0 , 5} \\
\operatorname{corr}\left(\mathrm{y}_{2}, \mathrm{z}_{5}\right)=\mathrm{c}_{52}=0,5\end{array}$ & $\begin{array}{l}1,2,3 \\
4,5\end{array}$ & $\begin{array}{l}\text { смысл } \quad\left(\mathrm{y}_{2}, \mathrm{z}_{1}\right)=\text { «лицо низкой } \\
\text { социальной активности», } \\
\text { смысл }\left(\mathrm{y}_{2}, \mathrm{z}_{2}\right)=\text { «низкая зарплата» } \\
\text { смысл }\left(\mathrm{y}_{2}, \mathrm{z}_{3}\right)=\text { порядочность } \\
\text { смысл } \quad\left(\mathrm{y}_{2}, \mathrm{z}_{4}\right)=\quad \text { «проявление } \\
\text { деспотизма» } \\
\text { смысл }\left(\mathrm{y}_{2}, \mathrm{z}_{5}\right)=\text { «честность» }\end{array}$ \\
\hline$\lambda_{3}=1,366273$ & $\begin{array}{l}\text { «обмануть, } \\
\text { чтобы...» }\end{array}$ & $\mathrm{y}_{3}$ & $\operatorname{Corr}\left(\mathrm{y}_{3}, \mathrm{z}_{1}\right)=\mathrm{c}_{13}=\mathbf{- \mathbf { 0 , 6 5 }}$ & 1 & $\begin{array}{l}\text { смысл } \quad\left(\mathrm{y}_{3}, \mathrm{z}_{1}\right)=\text { «лицо низкой } \\
\text { социальной активности» }\end{array}$ \\
\hline$\lambda_{4}=1$ & $\begin{array}{l}\text { «страху } \\
\text { нагнать» }\end{array}$ & $\mathrm{y}_{4}$ & $\begin{array}{l}\operatorname{corr}\left(\mathrm{y}_{4}, \mathrm{z}_{2}\right)=\mathrm{c}_{24}=\mathbf{0 , 5} \\
\operatorname{corr}\left(\mathrm{y}_{4}, \mathrm{z}_{3}\right)=\mathrm{c}_{34}=0,5 \\
\operatorname{corr}\left(\mathrm{y}_{4}, \mathrm{z}_{4}\right)=\mathrm{c}_{44}=\mathbf{- 0 , 5} \\
\operatorname{corr}\left(\mathrm{y}_{4}, \mathrm{z}_{5}\right)=\mathrm{c}_{54}=\mathbf{- \mathbf { 0 , 5 }}\end{array}$ & $\begin{array}{l}2,3 \\
4,5\end{array}$ & $\begin{array}{l}\text { смысл }\left(\mathrm{y}_{4}, \mathrm{z}_{2}\right)=\text { «низкая зарплата» } \\
\text { смысл }\left(\mathrm{y}_{4}, \mathrm{z}_{3}\right)=\text { антипорядочность } \\
\text { смысл } \quad\left(\mathrm{y}_{4}, \mathrm{z}_{4}\right)=\quad \text { «проявление } \\
\text { деспотизма» } \\
\text { смысл }\left(\mathrm{y}_{4}, \mathrm{z}_{5}\right)=\text { aнтичестность }\end{array}$ \\
\hline
\end{tabular}




\begin{tabular}{|c|c|c|c|c|c|c|}
\hline \multirow{4}{*}{ Impact Factor: } & ISRA (India) & $=3.117$ & SIS (USA) & $=0.912$ & ICV (Poland) & $=6.630$ \\
\hline & ISI (Dubai, UAI & $=0.829$ & РИНЦ (Russia & $=0.156$ & PIF (India) & $=1.940$ \\
\hline & GIF (Australia) & $=0.564$ & ESJI (KZ) & $=8.716$ & IBI (India) & $=4.260$ \\
\hline & JIF & $=1.500$ & SJIF (Morocce & $=5.667$ & OAJI (USA) & $=0.350$ \\
\hline
\end{tabular}

Таблица 8. Значения субъективных весов и коэффициент регрессии

\begin{tabular}{|l|l|l|l|}
\hline Номер j & $\begin{array}{l}\text { Экспертно } \\
\text { назначенный вес } \beta_{\mathrm{j}}\end{array}$ & $\begin{array}{l}\text { Формула } \\
\text { Преобразования } \beta_{\mathrm{j}} \text { в } \beta^{+}{ }_{\mathrm{j}}\end{array}$ & $\begin{array}{l}\text { Коэффициент } \\
\text { регрессии } \beta^{+}{ }_{\mathrm{j}}\end{array}$ \\
\hline 1 & 4 & $\beta^{+}{ }_{1}=0,368420804=\beta_{1} * \beta^{+}{ }_{2}$ & 0,368420804 \\
\hline 2 & 1 & $\beta^{+}{ }_{2}=0,092105201=\beta_{2} / \beta^{+}{ }_{2}$ & 0,092105201 \\
\hline 3 & 5 & $\beta^{+}{ }_{3}=0,460526005=\beta_{3} / \beta^{+}{ }_{2}$ & 0,460526005 \\
\hline 4 & 2 & $\beta^{+}{ }_{4}=0,184210402=\beta_{4} / \beta^{+}{ }_{2}$ & 0,184210402 \\
\hline 5 & 3 & $\beta^{+}{ }_{5}=0,276315603=\beta_{5} / \beta^{+}{ }_{2}$ & 0,276315603 \\
\hline
\end{tabular}

Валидной переменной $\mathrm{y}^{+}$(ее вес равен $0 \%$, $\left.\lambda_{5}=0.0000\right)$ нами вложен индикатор присутствия знания: $\mathrm{c}_{25}=0.5$, с использованием ОС3 2. Извлечем знание по ОМ МЛРА в $2,60 \%$ $\left(\beta^{+}=0.13012\right)$.

Смысл $\left(\mathrm{z}^{+}{ }_{6}, \beta_{5}=3\right)=3 *$ смысл $\left(\beta^{+}{ }_{5}\right)^{*}$ смысл $(\mathrm{y} 5, \mathrm{z} 5)=3 *[$ «честность»]=«честность». Мы извлекли, применяя ОМ МЛРА, еще 2,60\% знаний, внесенных применением ОС $32\left(\mathrm{c}_{25}=0.5\right)$. В итоге $100 \%=37,96 \%+21,07 \%+21,07 \%+17,29 \%+2,6 \%$ знаний мы когнитивно извлекли с применением математической модели и решив когнитивную Смысловую Обратную Задачу СОЗ.

\section{Заключение}

Мы рассмотрели новые моральные характеристики индивида, когнитивные ограничения морального типа, породили новые смыслы характеристикам морального климата, направлениям работ муниципального органа по мониторингу моральной среды человеческих ресурсов. новые моральные характеристики индивида мы рекомендуем применять на практике.

Они не учитываются в нормативных документах, при планировании этапов в проектах по модернизации сознания общества и каждого казахстанца. Новые смыслы характеристик морального поведения индивида делает актуальным предлагаемое рассмотрение вопросов (при реализации Программы «Рухани жангыру», в темах разделов поведенческой экономики.

Разработанная Когнитивная Модель Структуры Муниципального Органа по Мониторингу Моральной Среды для подвидов Человеческих Ресурсов основана на многих моделях () смотрите выше). Значимости и новизна моделей приведены в статье [1].

Мы рассмотрели некоторые нами измеряемые скрытые моральные качества (показатели), присущие, по нашему мнению, трудовым ресурсам стран СНГ. Индивиды, принадлежащие к группе человеческих ресурсов, используют такие «средства», что их смыслы аддитивно суммируются и когнитивно равны смыслу Смысл $\left(\mathrm{z}^{+}\right.$; «влияющие коэффициенты регрессии») $=$ «стремится обмануть, а не добросовестно сделать что-либо». Суммарные смыслы 5 влияющих коэффициентов регрессии равны следующим 5 «честная порядочность» (отличается от демонстративной порядочности), честная рабская антипорядочность, батрак, коррупция госслужащих (честная вынужденная антипорядочность), честность. Эти 5 смыслов когнитивно конструктированы из подмножеств 12 индивидуальных моральных показателей индивидов из Таблицы 7. Мы не вычисляли значения функций ценностей, как в статье [20], а нашли когнитивные смыслы скрытых показателей. Вопросы количественной ценности найденных показателей требует отдельного рассмотрения. Нами получен один пример сформированной структуры муниципального органа по мониторингу моральной среды. Когнитивная модель структуры муниципальных органов по мониторингу моральной среды для видов человеческих ресурсов состоит из 4-х департаментов, из 12 отделов, соответствующих своему 12 валидному (и моральному) показателю.

С учетом смыслов Z-переменных, имеющих 2 смысла, количество «узких» специалистов увеличится до 20. Для 12 или 20 специальностей существует 6 отделов, функционально обязанных с суммарными компетенциями той или иной части из вышеназванных отделов. Отделы должны иметь 6 документов «Положение об отделе...», где прописаны обязанности «узких» специалистов отдела с названием, отражающее суммарный смысл. Каждый смысл порождает свое название отделу, с соответствующими сотрудникам функциональными обязанностями.

Мы привели разные обоснования для учреждения 4-х Департаментов по мониторингу моральной среды человеческих ресурсов, в состав которых целесообразно включить 12 «узких» специалистов, сгруппированных по профилям из $2,5,1,4$ наименований. Веса Департаментов упорядочены в порядке их приоритетности: №1 (2 разных профиля, 37,96\%), №2 (5 разных профилей, 34.59\%), №3(один профиль, 34.157\%), №4 (4 разных профилей, 25\%,). Заметим, что мониторинг лиц, действующих по принципу 


\begin{tabular}{|c|c|c|c|c|c|c|}
\hline \multirow{4}{*}{ Impact Factor: } & ISRA (India) & $=3.117$ & SIS (USA) & $=0.912$ & ICV (Poland) & $=6.630$ \\
\hline & ISI (Dubai, UAI & $=0.829$ & РИНЦ (Russia & $=0.156$ & PIF (India) & $=1.940$ \\
\hline & GIF (Australia) & $=0.564$ & ESJI (KZ) & $=8.716$ & IBI (India) & $=4.260$ \\
\hline & JIF & $=1.500$ & SJIF (Morocce & $=5.667$ & OAJI (USA) & $=0.350$ \\
\hline
\end{tabular}

«обмануть, чтобы...» настолько много - 34.157\%, что вес Департамента №3 по их профилю сопоставима с Департаментом №2 (5 разных профилей, $34.59 \%$ ). Доля обманщиков сопоставима с долей «принудителей всех мастей» - необходимо когнитивно смоделировать подвиды профиля для специалиста из Департамента №3.

Специалисты разных профилей должны быть объединены в 5 отделов-по числу экспертных значений коэффициентов регрессии. Число специалистов одного профиля (из 12) должно быть кратно фактической нагрузке в отделе. Официальные названия департаментов, отделов обычно соответствуют концепции внутренней политики, мы даем лишь функциональные смыслы их направлениям работы. Вопросы нормирования труда каждого специалистов и вопросы разработки классификатора профессий рабочих и новых должностей служащих остаются вне рамок статьи.

\section{References:}

1. Zhanatauov, S. U. (2019). Soefficients of regression, containing mathematically introduced and cognitivelly extractabled knowledge. ISJ Theoretical \& Applied Science, 06 (74): 613-622.

2. Zhanatauov, S. U. (2018). Model of digitalization of indicators of individual consciousness. Int.Scien. Jour. "Theoretical \&Applied Science”. 2018, №6(62): 101-110. www.t-science.org

3. Zhanatauov, S. U. (2018). Inverse model of multiple linear regression analysis. ISJ Theoretical \&Applied Science. 2018, №4(60): 201-212. www.t-science.org

4. Zhanatauov, S. U. (2018). Soefficients of regression to help progress of banks. Int.Scien.Jour. "Theoretical \&Applied Science”. 2018, № 10(66): 515-532. www.t-science.org

5. Zhanatauov, S. U. (2012). Primenenie metoda lineynoy regressii dlya samootsenki riska bankrotstva banka. Finansovaya analitika: problemy i resheniya, 15(105) - 2012 aprel', 4043.

6. Zhanatauov, S. U. (2018). Modeling eigenvectors with given the values of their indicated components. Int. Scientific Journal Theoretical \&Applied Science.2018, №11(67), 107-119. www.t-science.org

7. Zhanatauov, S. U. (2018). Inverse spectral problem with indicated values of components of the eigenvectors. ISJ Theoretical \&Applied Science.2018, №11(67), 359-370. www.tscience.org

8. Zhanatauov, S. U. (2017). The optimization problem with linearized equations f-parameters (f1,f2,f3,f4,f5,f6)-spectrum. International scientific journal Theoretical \&Applied Science.2017,№11,vol.55, 251-267. www.tscience.org
9. Zhanatauov, S. U. (2018). Inverse spectral problem. ISJ Theoretical \&Applied Science.2018, №12(68), 101-112. www.tscience.org

10. Zhanatauov, S. U. (2017). The optimization problem with linearized equations f-parameters (f1,f2,f3,f4,f5,f6)-spectrum. International scientific journal Theoretical \&Applied Science. 2017,№11,vol.55, 251-267. www.t-science.org

11. Chalmers, C. P. (1975). Generation of correlation matrices with a given eigenstructure. J. Stat. Comp. Simul., 1975, vol.4, 133139.

12. Zhanatauov, S. U. (2013). Kognitivnaya karta i model' sotsial'no-ekonomicheskikh faktorov kar'ernoy uspeshnosti shkol'nikov munitsipal'nykh shkol SShA. Sibirskiy pedagogicheskiy zhurnal. 2013, №6, 28-33.

13. Zhanatauov, S. U. (2014). Analiz budushchikh debitorskoy i kreditorskoy zadolzhen nostey munitsipalitetov gorodov. Ekonomicheskiy analiz:teoriya i praktika. M.:№2(353), 54-62.

14. Zhanatauov, S. U. (2015, May 16-17). Kognitivnaya karta $i$ kognitivnaya model' analiza glavnykh komponent (telekommunikatsionnaya otrasl'). Natsional'naya assotsiatsiya uchenykh (NAU).IX Mezhd. nauch.-prakt. konf: «Otechestvennaya nauka v epokhu izmeneniy: postulaty proshlogo i teorii novogo vremeni». (pp.55-58). Rossiya, Ekaterinburg.

15. Zhanatauov, S. U. (2013). Obratnaya model' glavnykh komponent. (p.201). Almaty: Kazstatin-form.

16. Zhanatauov, S. U. (2019). A matrix of values the coefficients of combinational proportionality. Int. Scientific Journal Theoretical \&Applied Science.2019,71№3 (68), 401-419. www.tscience.org 


\begin{tabular}{llllll} 
& ISRA (India) $=\mathbf{3 . 1 1 7}$ & SIS (USA) $=\mathbf{0 . 9 1 2}$ & ICV (Poland) & $\mathbf{= 6 . 6 3 0}$ \\
Impact Factor: & ISI (Dubai, UAE) $=\mathbf{0 . 8 2 9}$ & PUHЦ (Russia) $=\mathbf{0 . 1 5 6}$ & PIF (India) & $=\mathbf{1 . 9 4 0}$ \\
& GIF (Australia) $=\mathbf{0 . 5 6 4}$ & ESJI (KZ) & $\mathbf{8 . 7 1 6}$ & IBI (India) & $\mathbf{4 . 2 6 0}$ \\
& JIF & $\mathbf{1 . 5 0 0}$ & SJIF (Morocco) $=\mathbf{5 . 6 6 7}$ & OAJI (USA) & $\mathbf{= 0 . 3 5 0}$ \\
\hline
\end{tabular}

17. Zhanatauov, S. U. (2018). Model of digitalization of the validity indicators and of the measurable indicators of the enterprise. Int.Scien.Jour. "Theoretical \&Applied Science". 2018, № 9(65): 315-334. www.t-science.org

18. Axelrod, R. (1976). Structure of Decision: the Cognitive Maps of Political Elites. Princeton.Univ.Press, pp. xvi +404 .
19. Zhanatauov S. U. (1988). Ofunktsional'nom napolnenii PPP "Spektr". Sistemnoe modelirovanie-13. (pp.3-11). Novosibirsk.

20. (2018). Digitalization of the behavioral model with errors of non-returnable costs. Scien.Jour. "Theoretical \&Applied Science". 2018, №8(64): 101-110. www.t-science.org 